\title{
Morse Theory and the topology of holomorphic foliations near an isolated singularity*
}

\author{
Beatriz Limón and José Seade \\ Instituto de Matemáticas, Unidad Cuernavaca \\ Universidad Nacional Autónoma de México
}

\begin{abstract}
Let $\mathcal{F}$ be the germ at $\mathbf{0} \in \mathbb{C}^{n}$ of a holomorphic foliation of dimension $d, 1 \leq d<n$, with an isolated singularity at $\mathbf{0}$. We study its geometry and topology using ideas that originate in the work of Thom concerning Morse theory for foliated manifolds. Given $\mathcal{F}$ and a real analytic function $g$ on $\mathbb{C}^{n}$ with a Morse critical point of index 0 at $\mathbf{0}$, we look at the corresponding polar variety $M=M(\mathcal{F}, g)$. These are the points of contact of the two foliations, where $\mathcal{F}$ is tangent to the fibres of $g$. This is analogous to the usual theory of polar varieties in algebraic geometry, where holomorphic functions are studied by looking at the intersection of their fibers with those of a linear form. Here we replace the linear form by a real quadratic map, the Morse function $g$. We then study $\mathcal{F}$ by looking at the intersection of its leaves with the level sets of $g$, and the way how these intersections change as the sphere gets smaller.
\end{abstract}

\section{Introduction}

In 1964 René Thom wrote a beautiful article [29] explaining how the classical ideas of Morse theory can be adapted to studying the topology of non-singular foliations on smooth manifolds. The purpose of this work is to adapt those ideas to studying the topology of holomorphic foliations near an isolated singular point.

We consider a holomorphic foliation $\mathcal{F}$ of dimension $d \geq 1$ defined on an open neighbourhood $\mathcal{U}$ of the origin $\mathbf{0} \in \mathbb{C}^{n}$, with a unique singular point at $\mathbf{0}$. To study its topology, we consider a function $g$ on $\mathcal{U}$ with a Morse critical point

* Research partially supported by PAPIIT-UNAM, CONACYT (Mexico) grants 55084 and 162340 , and by CNRS-CONACYT through the Laboratorio International Solomon Lefschetz (LAISLA).

Key-words: Holomorphic foliation, Morse function, Morse index, polar variety, contact point, gradient flow.

Mathematics Subject Classification. Primary: 32S65, 37F75 Secondary: 53C12, 58F18 
at $\mathbf{0}$ of index 0 , so that its non-critical levels are diffeomorphic to spheres; call these simply "spheres". Look at the restriction of $g$ to the leaves of $\mathcal{F}$. The critical points of $\left.g\right|_{\mathcal{L}}$ are the points of contact of $\mathcal{F}$ with the fibres of $g$, where the leaves of $\mathcal{F}$ are tangent to the fibres of $g$. The set of all such points is the polar variety of $\mathcal{F}$ relative to $g$, in analogy with this classical notion in algebraic geometry, where the role of $g$ is usually played by linear forms.

Inspired by the way how J. Milnor studied the topology of complex singularities in [21], we want to study the topology of $\mathcal{F}$ near $\mathbf{0}$ by looking at the intersection of the leaves of $\mathcal{F}$ with a sufficiently small sphere $\mathbb{S}_{\mathcal{E}}$, and the way how these intersections change as we make the radius of the sphere tend to 0 . In fact this point of view for studying holomorphic foliations already appears in [2, 5, 9, and this work is very much indebted those articles, specially to 9 .

Of course the contacts of the leaves of $\mathcal{F}$ with the fibres of $g$ can be degenerate. If the contacts are all non-degenerate, then we say that $\mathcal{F}$ carries a Morse structure compatible with $g$, or simply that $M$ carries the Morse structure of $g$, for short. In the sequel we give various examples of both, degenerate and non-degenerate contacts.

Our first result (Theorem 2.1) is the analogous in our setting of a classical result for polar varieties (see for instance [14]): we prove that the restriction of $g$ to the leaves of $\mathcal{F}$ is a Morse function on each leaf if and only if away from $\mathbf{0}$, the polar variety $M^{*}=M \backslash\{\mathbf{0}\}$ is a smooth, reduced, submanifold of $\mathbb{C}^{n}$ of codimension $2 d$ and $M^{*}$ is everywhere transversal to $\mathcal{F}$.

The topology of holomorphic foliations near a singular point can be rather complicated. In order to have a certain understanding of the behaviour of $\mathcal{F}$ near $\mathbf{0}$ we ask for the condition that the polar set $M=M(\mathcal{F}, g)$ be real analytic at $\mathbf{0}$. This happens in many interesting families, including all foliations of dimension or codimension one. In this case we say that the foliation is contact-analytic with respect to the Morse function $g$. We have (Theorem 3.3) that if $\mathcal{F}$ is contact-analytic and carries the Morse structure of $g$, then the corresponding polar variety has finitely many irreducible components, say $M_{1}, \ldots, M_{r}$, all of them pairwise disjoint away from $\mathbf{0}$; each of these is smooth away from $\mathbf{0}$, of real codimension $2 d$, transversal to the foliation and consists of points where the contacts have all the same Morse index.

Using this, we can give the following topological picture of $\mathcal{F}$ near $\mathbf{0}$ (Theorem 4.3): we equip $\mathcal{F}$ with the gradient flow on the leaves, i.e., the flow $\mathcal{G}_{\mathcal{F}}$ of the vector field on $\mathcal{U}$ obtained by projecting at each point the gradient of $g$ to the tangent space of $\mathcal{F}$. Let $\mathbb{B}_{\varepsilon}$ be a sufficiently small $g$-ball centred at $\mathbf{0}$. This ball splits in two disjoint $\mathcal{G}_{\mathcal{F}}$-invariant sets: the saturated $\widehat{M}$ of $M \cap \mathbb{B}_{\varepsilon}$ by $\mathcal{F}$ and its complement $K:=\mathbb{B}_{\varepsilon} \backslash \widehat{M}$. On $K$ the topology is somehow simple and the dynamics can be rich, while on $\widehat{M}$ the topology is rich and the dynamics is often simpler.

Each leaf $\mathcal{L}$ in $K$ is homeomorphic to a product $\left(\mathcal{L} \cap \mathbb{S}_{\varepsilon}\right) \times \mathbb{R}$, immersed in $\mathbb{C}^{n}$ so that it is transversal to each $g$-sphere around $\mathbf{0}$ and the $\alpha$-limit of each orbit in $K$ of the gradient flow $\mathcal{G}_{\mathcal{F}}$ is the origin $\mathbf{0}$. Thus, for each positive number $\varepsilon^{\prime}<\varepsilon$, the leaves of $\mathcal{F}$ in $K$ meet the $g$-sphere $\mathbb{S}_{\mathcal{\varepsilon}^{\prime}}$ transversally and 
define a real analytic foliation on it, which can have very rich dynamics. For instance [5], if $\mathcal{F}$ is defined by a linear vector field in the Poincaré domain with generic eigenvalues, then the foliation on $\mathbb{S}_{\varepsilon^{\prime}}$ actually is defined by a flow which is Morse-Smale.

The picture on $\widehat{M}$ is rather different: the $\alpha$-limit of $\mathcal{G}_{\mathcal{F}}$ of each leaf in $\widehat{M}$ is the set of points where the corresponding leaf meets the polar variety $M$. The intersection of the leaves of $\mathcal{F}$ with $M$ is always transverse, as described above, and the topology of every leaf $\mathcal{L} \subset \widehat{M}$ is determined by its intersection with the boundary sphere, $\mathcal{L} \cap \mathbb{S}_{\varepsilon}$, and the points where $\mathcal{L}$ meets $M$ : each such intersection point comes with a Morse index, that tells us what type of handle we must attach to the leaf when passing through that point. In particular, if $\mathcal{L}$ is compact, then its Euler-Poincaré characteristic $\chi(\mathcal{L})$ equals the number of intersection points in $\mathcal{L} \cap M$, counted with sign. The sign is negative when the corresponding Morse index is odd, and positive otherwise.

For instance, for generic linear actions of $\mathbb{C}^{m}$ in $\mathbb{C}^{n}$ in the Siegel domain (see [19] and Example 1.5 below), $m<<n$, each Siegel leaf $\mathcal{L}$ is a copy of $\mathbb{C}^{m}$ embedded in $\mathbb{C}^{n}$ with a unique point of minimal distance to $\mathbf{0}$. The gradient flow of the quadratic form $\left(z_{1}, \cdots, z_{n}\right) \mapsto\left|z_{1}\right|^{2}+\cdots+\left|z_{n}\right|^{2}$ describes each Siegel leaf as a cylinder over its intersection with a sphere, $\mathcal{L} \cap \mathbb{S}^{2 n-1} \cong \mathbb{S}^{2 m-1}$, to which we attach a handle of Morse index 0, i.e., a $2 m$-disc.

We remark that the topology of the polar varieties that arise in this way can be rather interesting. In fact these are all manifolds with a canonical complex structure determined by a foliated atlas for $\mathcal{F}$ (see Remark 2.5), and with a rich geometry. For instance, when $\mathcal{F}$ is the foliation of a linear flow in the Siegel domain with generic eigenvalues and $g$ is the usual metric, the manifold $M_{\varepsilon}=M \cap \mathbb{S}_{\varepsilon}$ is the space of Siegel leaves of the flow and it has very interesting topology [5, 16. This type of manifolds has been studied by several authors giving rise to the theory of $L V-M$ manifolds (see for instance [17, 19, 20]). These belong also to an interesting class of manifolds that appear in mathematical physics and algebraic topology, called moment-angle manifolds (see for instance [8]). On the other hand, the polar varieties associated to non-linear vector fields in $\mathbb{C}^{n}$ of the form $\left(\lambda_{1} z_{\sigma(1)}^{a_{1}}, \cdots, \lambda_{n} z_{\sigma(n)}^{a_{n}}\right)$, where the $\lambda_{i}$ are non-zero complex numbers, $a_{i} \geq 2$, and $\sigma$ is a permutation of the set $\{1, \cdots, n\}$, have been studied in [24, 25, 26] in relation with Milnor fibrations for real singularities. In all these cases the function that defines the polar variety determines a Milnor open-book 21. The study of this type of singularities has evolved and several related lines of research are being pursued by various authors, see for instance [4, 6, 7, 22,

Notice that if $\mathbb{S}_{\mathcal{\varepsilon}}$ is a small $g$-sphere in $\mathcal{U}$ around $\mathbf{0}$, then the intersection of the leaves of $\mathcal{F}$ with $\mathbb{S}_{\varepsilon}$ defines a real analytic foliation $\mathcal{F}_{\varepsilon}$ on the sphere, which is singular at $M_{\varepsilon}:=\mathbb{S}_{\varepsilon} \cap M$, where $M$ is the polar variety. Away from $M_{\varepsilon}$ the leaves of $\mathcal{F}_{\varepsilon}$ have real dimension $2 d-1$. In Section 5 we address the problem of studying how the topology of the foliation $\mathcal{F}_{\varepsilon}$ varies as $\varepsilon \rightarrow 0$. For instance if $M=\{\mathbf{0}\}$, as it happens for linear vector fields in the Poincaré domain, then the topology of $\mathcal{F}_{\varepsilon}$ is independent of $\varepsilon$. This happens also for generic flows in 
the Siegel domain, and more generally for generic linear $\mathbb{C}^{m}$-actions on $\mathbb{C}^{n}$ in the Siegel domain. In 9 it was proved that if $\mathcal{F}$ has dimension 1 and all the points in $M$ have Morse index 0, i.e., they correspond to local minimal points in their leaves, then the topology of $\mathcal{F}_{\varepsilon}$ is independent of $\varepsilon$. Here we show that the same statement is true for foliations of arbitrary dimension (Theorem 5.1).

Finally, Section 6 is motivated by [12, where the authors prove that every linear foliation $\mathcal{F}$ in $\mathbb{C}^{n}$ has associated an invertible symmetric $n \times n$ complex matrix, and the set of all such matrices that define a foliation that carries the Morse structure of $Q=\left|z_{1}\right|^{2}+\left|z_{2}\right|^{2}+\cdots+\left|z_{n}\right|^{2}$ is dense. In fact their complement is a Zariski closed proper subset. Here we look at homogeneous foliations of degree $k>1$ and show that up to isotopy, all these carry the Morse structure of the usual hermitian metric in $\mathbb{C}^{n}$.

\section{Some definitions and examples}

Recall that if $M$ is a smooth $\left(=C^{\infty}\right)$ manifold and $g: M \rightarrow \mathbb{R}$ is a smooth function with isolated critical values, then $g$ is a Morse function if its critical points are all non-degenerate. Usually it is also asked that different critical points correspond to distinct critical values; yet, in this article we ignore that condition. The Morse lemma says that in a neighbourhood of a critical point, one can choose a coordinate chart such that $g$ takes locally the form:

$$
g\left(x_{1}, \ldots, x_{k}, \ldots, x_{m}\right)=-x_{1}^{2}-\ldots-x_{k}^{2}+x_{k+1}^{2}+\ldots .+x_{m}^{2},
$$

where $m$ is the dimension of $M$. The number $k$ of negative signs is called the Morse index of $g$ at the critical point. Points of Morse index 0 or $m$ correspond to local minimal and maximal points respectively. All others are saddle-points.

In this work $M$ is a neighbourhood $\mathcal{U}$ of the origin $\mathbf{0}$ in $\mathbb{C}^{n}, n \geq 2$, and $g: \mathcal{U} \rightarrow \mathbb{R}$ is a Morse function with a unique critical point at $\mathbf{0}$ of Morse index 0 . Its level surfaces near $\mathbf{0}$ are diffeomorphic to $(2 n-1)$-spheres.

The following concept is introduced in [9] for 1-dimensional foliations under the name of foliations of Morse-type. See also 24] where these are studied in relation with finite determinacy of map-germs, and also [12, 13] where the concept is studied for certain codimension 1 foliations. We find that the name "Morse-type foliation" can be confused with "Morse foliation" (these are real codimension 1 foliations which are locally defined by a Morse function). Thus we use a different name here.

Unless stated otherwise, throughout this article $\mathcal{F}$ is a holomorphic foliation of dimension $d \geq 1$ defined on $\mathcal{U}$, with a unique singular point at $\mathbf{0}$, and $g$ is a Morse function on $\mathcal{U}$ with a unique critical point at $\mathbf{0}$ of Morse index 0 . (See [3, 27] for basic material on holomorphic foliations.) A leaf of $\mathcal{F}$ means a leaf on $U \backslash\{\mathbf{0}\}$.

Definition 1.1. We say that $\mathcal{F}$ has a Morse structure compatible with that of $g$ if the restriction $g_{\mathcal{L}}$ of $g$ to each leaf $\mathcal{L}$ of $\mathcal{F}$, is a Morse function on $\mathcal{L}$. For the sake of "compactness", in the above situation we will often say that $\mathcal{F}$ carries the Morse structure of $g$. 
The lemma below follows easily from [29] and its proof is left to the reader:

Lemma 1.2. The critical points of $g_{\mathcal{L}}$ are the points where $\mathcal{L}$ is tangent to the level surfaces of $g$.

Denote by $M=M(\mathcal{F}, g)$ the set of points in $\mathcal{U}$ where the two foliations are tangent. Lemma 1.2 says that these are exactly the critical points of the restrictions of $g$ to the leaves of $\mathcal{F}$. These are the points of contact of the two foliations in the notation of Thom 29. We assume, by definition, that this set contains $\mathbf{0}$, since at this point both foliations are singular and their tangent spaces are 0-dimensional.

Definition 1.3. We call $M:=M(\mathcal{F}, g)$ the polar set of $\mathcal{F}$ with respect to the Morse function $g$.

For each point $\mathbf{z} \in \mathcal{U} \backslash\{\mathbf{0}\}$, let $\mathcal{L}_{\mathbf{z}}$ be the leaf of $\mathcal{F}$ that contains $\mathbf{z}$ and $T_{\mathbf{z}} \mathcal{L}_{\mathbf{z}}$ its tangent space. Then $M^{*}:=M \backslash\{\mathbf{0}\}$ is:

$$
M^{*}:=\left\{\mathbf{z} \in \mathcal{U} \subset \mathbb{C}^{n} \mid \operatorname{Re}\langle v, \nabla g(\mathbf{z})\rangle=0, \quad \forall v \in T_{\mathbf{z}} \mathcal{L}_{\mathbf{z}}\right\},
$$

where $\nabla g$ is the gradient vector field and $\langle$,$\rangle is the usual hermitian product,$ whose real part is the usual inner product in $\mathbb{R}^{2 n} \cong \mathbb{C}^{n}$.

Notice that at each point $\mathbf{z} \in \mathcal{L}$ we have a vector $\nabla g_{\mathcal{L}}(\mathbf{z}) \in T_{\mathbf{z}} \mathcal{L}_{\mathbf{z}}$, which is the projection to $T_{\mathbf{z}} \mathcal{L}_{\mathbf{z}}$ of the gradient vector $\nabla g(\mathbf{z})$. In fact $\nabla g_{\mathcal{L}}(\mathbf{z}) \in T_{\mathbf{z}} \mathcal{L}_{\mathbf{z}}$ is the gradient of the restriction of $g$ to the leaf $\mathcal{L}_{\mathbf{z}}$. We thus have a smooth vector field $\nabla g_{\mathcal{F}}$ on $\mathcal{U}$, which is everywhere tangent to the leaves of $\mathcal{F}$.

Let $\mathcal{G}_{\mathcal{F}}$ be the (local) flow of $\nabla g_{\mathcal{F}}$. We call this the gradient flow on the leaves of $\mathcal{F}$ for the function $g$, in analogy with 9 . This flow leaves invariant each leaf of $\mathcal{F}$, and at each point moves along its leaf in the direction that (travelling backwards) brings it "closer" to $\mathbf{0}$ with respect to the level sets of the Morse function $g$.

The fixed points of $\mathcal{G}_{\mathcal{F}}$, others than $\mathbf{0}$, are the points where $\nabla g_{\mathcal{L}}(\mathbf{z})$ is orthogonal to $T_{\mathbf{z}} \mathcal{L}_{\mathbf{z}}$. These are exactly the points of contact of $\mathcal{F}$ with the fibres of $g$. Hence we have:

Lemma 1.4. The set $M$ of points where $\mathcal{F}$ is tangent to the level hypersurfaces of $g$ is the singular set (fixed points) of the gradient flow $\mathcal{G}_{\mathcal{F}}$. Moreover, $\mathcal{F}$ carries the Morse structure of $g$ if and only if the singularities of $\mathcal{G}_{\mathcal{F}}$ on each leaf are isolated and non-degenerate.

Examples 1.5. In [5] the authors study the topology of flows defined by a linear, diagonal, vector field $F$ in $\mathbb{C}^{n}$ with eigenvalues $\Lambda=\left\{\lambda_{1}, \ldots, \lambda_{n}\right\}$. From the viewpoint of dynamics, maybe the most interesting case is when $F$ is in the Poincaré domain, i.e., the convex hull $\mathcal{H}\left(\lambda_{1}, \ldots, \lambda_{n}\right)$ does not contain the origin $0 \in \mathbb{C}$. In this case one has that every leaf is transverse to every sphere around the origin $\mathbf{0} \in \mathbb{C}^{n}$ and has $\mathbf{0}$ in its closure. Therefore, topologically, the leaves are all cylinders over their intersection with a small sphere $\mathbb{S}_{\varepsilon}$ around $\mathbf{0}$, i.e., 
they are of the form $\left(\mathcal{L} \cap \mathbb{S}_{\varepsilon}\right) \times \mathbb{R}$. Hence they are immersed copies of either $\mathbb{C}$ or $\mathbb{C}^{*}$. This is used in [5] to prove the structural stability of this type of foliations.

On the other hand, if 0 is in $\mathcal{H}\left(\lambda_{1}, \ldots, \lambda_{n}\right)$ we say that $F$ is in the Siegel domain. In this case if the eigenvalues are "generic enough" then by 5 there is an open dense set $\widehat{M}$ in $\mathbb{C}^{n}$ of points that belong to a Siegel leaf. These leaves are embedded copies of $\mathbb{C}$ which have a unique point of minimal distance to $\mathbf{0}$.

The space $M^{*}$ of Siegel leaves is a real analytic submanifold of $\mathbb{C}^{n}$ of real codimension 2 defined by $M^{*}=M \backslash\{\mathbf{0}\}$, where $M$ is given by the equations:

$$
\langle F(\mathbf{z}), \mathbf{z}\rangle:=\sum_{i=1}^{n} \lambda_{i}\left|z_{i}\right|^{2}=0
$$

The topology and geometry of this type of manifolds has been investigated by Lopez de Medrano, Verjovsky and Meersseman, obtaining remarkable results (see for instance [20] and the bibliography on it). Following [19], consider now $m$ linear vector fields in $\mathbb{C}^{n}, n>2 m, F_{j}=\left(\lambda_{1}^{j} z_{1}, \ldots, \lambda_{n}^{j} z_{n}\right), j=1, \ldots, m$. For each $k=1, \ldots, n$, set $\Lambda_{k}=\left(\lambda_{k}^{1}, \ldots, \lambda_{k}^{m}\right)$, so we have $n$ vectors in $\mathbb{C}^{m}$. Assume these vector satisfy:

a) The Siegel condition: $\mathbf{0} \in \mathcal{H}\left(\Lambda_{1}, \ldots, \Lambda_{n}\right)$. That is, the origin $\mathbf{0} \in \mathbb{C}^{m}$ is in the convex hull of the $n$ points in $\mathbb{C}^{m}$ determined by the $\Lambda_{i}$; and

b) The weak hyperbolicity condition: no hyperplane through any $2 m$ of these $n$ points contains $\mathbf{0 .}$

Meersseman shows that the corresponding linear action of $\mathbb{C}^{m}$ on $\mathbb{C}^{n}$ has an open dense set of Siegel leaves: copies of $\mathbb{C}^{m}$ embedded with a unique point of minimal distance. These points of minimal distance parameterize the space of Siegel leaves, which is a smooth real submanifold of $\mathbb{C}^{n}$ of real codimension $2 m$, with a rich geometry and topology.

These are all examples of foliations that carry the Morse structure of the function on $\mathbb{C}^{n}$ given by $\left(z_{1}, \cdots, z_{n}\right) \stackrel{Q}{\mapsto}\left|z_{1}\right|^{2}+\cdots\left|z_{n}\right|^{2}$.

Recall that every point in $M$ is a critical point of the restriction $g_{\mathcal{L}}$ of $g$ to the corresponding leaf.

Definition 1.6. Assume that a point $\mathbf{z} \in M^{*}:=M \backslash\{\mathbf{0}\}$ is a non degenerate contact. Then define its Morse index relative to $\mathcal{F}$ to be the Morse index at $\mathbf{z}$ of the restriction of $g$ to the leaf that contains $\mathbf{z}$.

Since the leaves have real dimension $2 d$, a priori the possible Morse indices go from 0 to $2 d$. However, not all these indices are actually possible, because the fact that the foliation is holomorphic imposes certain restrictions. In fact just as in 21] or in [1, it can be shown that there are no critical points in the leaves of index greater than $d$.

Examples 1.7. i) Given integers $p, q>2$, let $F$ be the vector field defined by

$$
F\left(z_{1}, z_{2}\right)=\left(q z_{2}^{q-1}, p z_{1}^{p-1}\right) .
$$


Its integral lines are the level curves of the map $\left(z_{1}, z_{2}\right) \stackrel{f}{\mapsto}\left(z_{1}^{p}-z_{2}^{q}\right)$. In these examples the above function $Q$ is Morse restricted to each leaf. There is one separatrix, $V:=\left\{z_{1}^{p}=z_{2}^{q}\right\}$, which is transversal to all the spheres around $\mathbf{0}$, and all other fibres meet the $z_{1}$-axis at $p$ points and they have $q$ points on the $z_{2}$-axis. A straightforward computation shows that these are all points with Morse index 0 . There is a third component of the polar variety $M$, which is a real analytic surface with an isolated singularity at $\mathbf{0}$ :

$$
M^{-}:=\left\{\left(z_{1}, z_{2}\right) \in \mathbb{C}^{2} \mid q z_{2}^{q-1} \bar{z}_{1}=-p z_{1}^{p-1} \bar{z}_{2} ; z_{1}, z_{2} \neq 0\right\} .
$$

Each leaf has $p q$ saddles in this surface. As a matter of fact, one can easily check that $M^{-}$is ambient homeomorphic to the separatrix $V$. That is, there is a homeomorphism of $\mathbb{C}^{2}$ carrying $M^{-}$into $V$. We do not know whether this is a coincidence or a special case of a general theorem.

ii) Consider now the vector field $F\left(z_{1}, z_{2}\right)=\left(q z_{2}^{q-1}, 2 z_{1}\right), q>2$. Asides from the separatrix $V=\left\{z_{1}^{2}=z_{2}^{q}\right\}$, each leaf sufficiently near $V$ has 2 points of minimal distance in the $z_{1}$-axis and $q$ saddles in the $z_{2}$-axis. When $|t|=\left(\frac{2}{q}\right)^{\frac{q}{q-2}}$ the leaves $f^{-1}(t)$ have degenerate contacts. This happens because a new component of $M$ appears, this is the singular variety

$$
M^{-}=\left\{q z_{2}^{q-1} \bar{z}_{1}+z_{1} \bar{z}_{2}=0 ; z_{1}, z_{2} \neq 0\right\},
$$

which does not pass through $\mathbf{0}$, so we do not see it when we are near the origin. As we consider leaves which are further away from $V$, each leaf has 2 minimal points in the $z_{1}$-axis, as before, but they now have $q$ minimal points in the $z_{2}$-axis and new singularities appear: each leaf has $2 \mathrm{q}$ saddles along the surface $M^{-}$. Thence we see that even though all fibres $f^{-1}(t)$ are diffeomorphic for $t \neq 0$, yet, the way how they are embedded in $\mathbb{C}^{2}$ bifurcates as we pass from fibers inside the Milnor tube of radius $\left(\frac{2}{q}\right)^{\frac{q}{q-2}}$, to fibers outside this tube.

iii) For the linear vector field $\left(-z_{2}, z_{1}\right)$, whose integral lines are the fibers of the map $z_{1}^{2}+z_{2}^{2}$, the polar variety is a set of real dimension three and each leaf $\mathcal{L}=f^{-1}(t)$ has a curve of critical points, all of them degenerated contacts with a sphere. Yet, in 12 is proved that every linear foliation can be arbitrarily approximated by linear foliations having only non-degenerate contacts (see Section 6 below).

We remark that the same type of observations done above for foliations defined by a polynomial $f=z_{1}^{2}-z_{2}^{q}, q>2$, apply also in higher dimensions to all foliations defined by Pham-Brieskorn polynomials of the form:

$$
f\left(z_{1}, \cdots, z_{n}\right)=z_{1}^{2}+z_{2}^{a_{2}}+\cdots+z_{n}^{a_{n}}, \quad a_{i}>2 .
$$

This was first observed in [13] for certain cases. 


\section{Generic contacts and smoothness of the polar variety}

Our first result is the analogous in our setting of a classical result for polar varieties (compare with [14, 15]):

Theorem 2.1. Let $\mathcal{F}$ and $g: \mathcal{U} \rightarrow \mathbb{R}$ be as above, $\mathcal{U} \subset \mathbb{C}^{n}$. Let $M$ be the polar variety of $\mathcal{F}$ relative to $g$ and $M^{*}:=M \backslash\{\mathbf{0}\}$. Then $\mathcal{F}$ carries the Morse structure of $g$ if and only if $M^{*}$ is a smooth, reduced submanifold of $\mathbb{C}^{n}$ of codimension $2 d$ and $M^{*}$ is everywhere transversal to $\mathcal{F}$.

The following lemma proves one side of Theorem 2.1.

Lemma 2.2. If $\mathcal{F}$ carries the Morse structure of $g$, then $M^{*}$ is a smooth, reduced, real submanifold of $\mathbb{C}^{n}$ of codimension $2 d$ and $M^{*}$ is transversal to $\mathcal{F}$ everywhere.

Proof. Let $\mathbf{z} \neq \mathbf{0}$ in $\mathcal{U}$ and consider a foliated chart $U^{\prime}$ around $\mathbf{z}$ with holomorphic coordinates $\left(z_{1}, z_{2}, \ldots, z_{n}\right)$, such that the leaves of $\mathcal{F}$ are locally given by:

$$
\left\{z_{d+1}=c_{d+1}, \ldots, z_{n}=c_{n}\right\}
$$

where the $c_{i}$ are constants. Thus we can consider $g_{\mathcal{L}_{\mathbf{z}}}$ in $U^{\prime}$ as a function of $\left(z_{1}, \ldots, z_{d}\right)$, or equivalently of the real coordinates $\left(z_{1}, \bar{z}_{1}, \ldots, z_{d}, \bar{z}_{d}\right)$. Then the Hessian of $g_{\mathcal{L}_{\mathbf{z}}}$ at $\mathbf{z}$ looks like

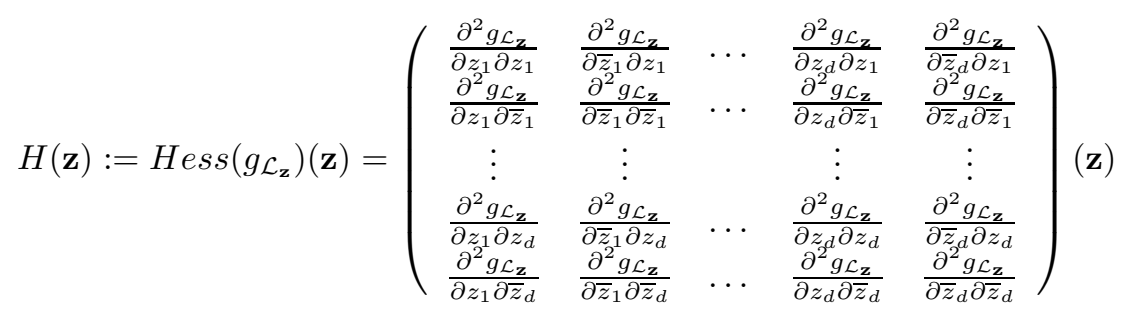

Define $G_{j, 0}(\mathbf{z}):=\frac{\partial}{\partial z_{j}} g_{\mathcal{L}_{\mathbf{z}}}(\mathbf{z})$ and $G_{0, j}(\mathbf{z}):=\frac{\partial}{\partial \bar{z}_{j}} g_{\mathcal{L}_{\mathbf{z}}}(\mathbf{z})$ for $1 \leq j \leq d$. Set

$$
G(\mathbf{z})=\left(G_{1,0}(\mathbf{z}), G_{0,1}(\mathbf{z}), \ldots G_{d, 0}(\mathbf{z}), G_{0, d}(\mathbf{z})\right) .
$$

Then $M$ is locally the zero set of the functions $\left\{G_{j, 0}(\mathbf{z}), G_{0, j}(\mathbf{z})\right\}_{1 \leq j \leq d}$ and their jacobian is

$$
D_{\mathbf{z}} G=\left(\begin{array}{cccccccc}
\frac{\partial G_{1,0}}{\partial z_{1}} & \frac{\partial G_{1,0}}{\partial \bar{z}_{1}} & \ldots & \frac{\partial G_{1,0}}{\partial z_{d}} & \frac{\partial G_{1,0}}{\partial \bar{z}_{d}} & \frac{\partial G_{1,0}}{\partial z_{d+1}} & \ldots & \frac{\partial G_{1,0}}{\partial \bar{z}_{n}} \\
\frac{\partial G_{0,1}}{\partial z_{1}} & \frac{\partial G_{0,1}}{\partial \bar{z}_{1}} & \ldots & \frac{\partial G_{0,1}}{\partial z_{d}} & \frac{\partial G_{0,1}}{\partial \bar{z}_{d}} & \frac{\partial G_{0,1}}{\partial z_{d+1}} & \ldots & \frac{\partial G_{0,1}}{\partial \bar{z}_{n}} \\
\vdots & \vdots & & \vdots & \vdots & \vdots & & \vdots \\
\frac{\partial G_{d, 0}}{\partial z_{1}} & \frac{\partial G_{d, 0}}{\partial \bar{z}_{1}} & \ldots & \frac{\partial G_{d, 0}}{\partial z_{d}} & \frac{\partial G_{d, 0}}{\partial \bar{z}_{d}} & \frac{\partial G_{d, 0}}{\partial z_{d+1}} & \ldots & \frac{\partial G_{d, 0}}{\partial \bar{z}_{n}} \\
\frac{\partial G_{0, d}}{\partial z_{1}} & \frac{\partial G_{0, d}}{\partial \bar{z}_{1}} & \ldots & \frac{\partial G_{0, d}}{\partial z_{d}} & \frac{\partial G_{0, d}}{\partial \bar{z}_{d}} & \frac{\partial G_{0, d}}{\partial z_{d+1}} & \ldots & \frac{\partial G_{0, d}}{\partial \bar{z}_{n}}
\end{array}\right)
$$


Observe that the submatrix formed by the first $2 d$ columns of $D_{\mathbf{z}} G(\mathbf{z})$ is the matrix $H(\mathbf{z})$. Since $\mathcal{F}$ carries the Morse structure of $g, D_{\mathbf{z}} G(\mathbf{z})$ has maximal rank $2 d$; therefore $M^{*}$ is defined locally as the inverse image of a regular value of the functions $\left\{G_{j, 0}(\mathbf{z}), G_{0, j}(\mathbf{z})\right\}_{1 \leq j \leq d}$. Hence it is a reduced real submanifold of codimension $2 d$.

On the other hand, consider the vectors $v_{j}=(0, \ldots, 0,1,0, \ldots, 0)$ which correspond to the canonical basis of $\mathbb{R}^{2 n}$ with respect to the coordinate system $\left(z_{1}, \bar{z}_{1}, \ldots, z_{n}, \bar{z}_{n}\right)$. The set $\left\{v_{j}\right\}_{1 \leq j \leq 2 d}$ forms a basis of the space $T_{\mathbf{z}} \mathcal{L}_{\mathbf{z}}$ for all $\mathbf{z}$ in $M^{*} \cap U^{\prime}$. If we apply $D_{\mathbf{z}} G$ to the vector $v_{j}$ we see it equals the $j$ th column of the matrix $D_{\mathbf{z}} G$. Since $D_{\mathbf{z}} G$ has rank $2 d$ we have that the tangent space $T_{\mathbf{z}} \mathcal{L}_{\mathbf{z}}$ is a direct sum complement of the kernel of $D_{\mathbf{z}} G$. Moreover the intersection between $T_{\mathbf{z}} \mathcal{L}_{\mathbf{z}}$ and $\operatorname{ker}\left(D_{\mathbf{z}} G\right)$ is trivial. Hence $T_{\mathbf{z}} \mathcal{L}_{\mathbf{z}}$ and $M^{*}$ have a transversal intersection.

The lemma below completes the proof of Theorem 2.1;

Lemma 2.3. Let $M^{*}$ be a real submanifold of $\mathbb{C}^{n}$ of codimension $2 d$, reduced, and assume $M^{*}$ is transversal to $\mathcal{F}$ everywhere. Then $\mathcal{F}$ carries the Morse structure of $g$.

Proof. Let us take $\left(z_{1}, \bar{z}_{1}, \ldots, z_{n}, \bar{z}_{n}\right)$ and $G$ as in the proof of the previous lemma. Recall $M=G^{-1}(0)$. By hypothesis, $M^{*}$ is a reduced, real submanifold of dimension $2(n-d)$ in $\mathbb{R}^{2 n}$, hence $D_{\mathbf{z}} G$ has rank $2 d$.

Assume $\mathbf{v}_{0}$ in $\mathbb{R}^{2 d}$ is different from zero and define $\mathbf{w}_{0}:=\left(\mathbf{v}_{0}, \mathbf{0}\right)$ in $\mathbb{R}^{2 n}$ for an appropriate coordinate chart as above. Then, as before, $\mathbf{w}_{0}$ is an element of $T_{\mathbf{z}} \mathcal{L}_{\mathbf{z}}$ and the matrices $D_{\mathbf{z}} G$ and $H(\mathbf{z})$ are related by $D_{\mathbf{z}} G=(H(\mathbf{z}), A)$, where $A$ is a real matrix of $2 d \times 2(n-d)$; thus, $D_{\mathbf{z}} G\left(\mathbf{w}_{0}\right)=H(\mathbf{z})\left(\mathbf{v}_{0}\right)$. By hypothesis, the spaces $\mathcal{L}_{\mathbf{z}}$ and $M^{*}$ have a transversal intersection. Thus the vector $\mathbf{w}_{0}$ is not in the kernel of $D_{\mathbf{z}} G$, for dimensional reasons. Hence $H(\mathbf{z})\left(\mathbf{v}_{0}\right)$ is different from zero. Since this happens for all $\mathbf{v}_{0}$ not equal to zero in $\mathbb{R}^{2 d}$, we have that $H(\mathbf{z})$ is non singular. Therefore $g_{\mathcal{L}_{\mathbf{z}}}$ is non-degenerate.

Remark 2.4. Usually, when we speak of "submanifolds" in differential topology we mean "reduced submanifolds". Yet, the example below shows that this can be misleading and one must take care of the algebra behind the screen. This is why we added the word "reduced" in the statement of Theorem 2.1.

Consider the foliation on $\mathbb{R}^{2}$ given by the fibers of the map $(x, y) \mapsto x^{4}+y$. Its leaves look like parabolas, but not quite. Consider now the contacts of this foliation with the foliation given by the lines $\{y=$ constant $\}$. Then the polar variety is

$$
M=\left\{(x, y) \in \mathbb{R}^{2} \mid x^{3}=0\right\} .
$$

So this is just the $y$-axis, as a set, which of course is a smooth submanifold of $\mathbb{R}^{2}$. Yet, in this case the $y$-axis comes with multiplicity 3 ; it is the preimage of the critical value 0 of the map $x^{3}$ in $\mathbb{R}^{2}$, and the contacts are all degenerate. 
Remark 2.5. In [10] Haefliger noticed that if $X$ is a complex manifold, of dimension say $n, \xi$ is a holomorphic regular foliation (i.e., with no singularities) of dimension $d, 1 \leq d<n$, and $N$ is a smooth differentiable submanifold of $X$ of real dimension $2 n-2 d$, which is everywhere transversal to $\xi$, then a foliated atlas for $\xi$ determines a canonical complex structure for $M$. This comes from the fact that a holomorphic foliated atlas for $\xi$ can be given by a locally finite collection $\left(U_{\alpha}, \Sigma_{\alpha}, p_{\alpha}\right)$ satisfying certain compatibility conditions, where the $U_{\alpha}$ form an open covering, the $\Sigma_{\alpha}$ are discs of dimension $2 n-2 d$ in $X$, transversal to $\xi$, and the $h_{\alpha}$ are local submersions $U_{\alpha} \rightarrow \Sigma_{\alpha}$ whose fibres give the plaques of $\xi$. Since the manifold $N$ is transversal to $\xi$, we can take the discs $\Sigma_{\alpha}$ as forming an atlas for $N$, and therefore the transversally holomorphic structure of $\xi$ determines a holomorphic structure for $N$. Hence if $\mathcal{F}$ is as above and it carries the Morse structure of $g$, then the polar variety $M^{*}$ inherits from $\mathcal{F}$ a canonical complex structure.

Remark 2.6. Notice that the previous discussion holds with minor modifications for Morse functions on $\mathcal{U}$ with arbitrary Morse index at $\mathbf{0}$. We have restricted the discussion to functions with Morse index 0 because their level surfaces are spheres that bound a compact region around $\mathbf{0}$, and we are interested in studying the topological and geometric behaviour of holomorphic foliations near $\mathbf{0}$.

Example 2.7. Consider the vector field $F(\mathbf{z})=\left(\lambda_{1} z_{\sigma(1)}^{a_{\sigma(1)}}, \ldots, \lambda_{n} z_{\sigma(n)}^{a_{\sigma(n)}}\right)$, where $\sigma$ is a permutation of $\{1, \ldots, n\}$ and $a_{k} \geq 2$ for all $k$. Let $\mathcal{F}$ be the foliation defined by $F$ and $Q(\mathbf{z})=\left|z_{1}\right|^{2}+\left|z_{2}\right|^{2}+\cdots+\left|z_{n}\right|^{2}$. The corresponding polar variety $M=M(\mathcal{F}, Q)$ is defined by the function:

$$
f\left(z_{1}, \cdots, z_{n}\right)=\lambda_{1} z_{\sigma(1)}^{a_{\sigma(1)}} \bar{z}_{1}+\cdots+\lambda_{n} z_{\sigma(n)}^{a_{\sigma(n)}} \bar{z}_{n} .
$$

In 25] is proved that this function has $\mathbf{0}$ as its only critical point, so $M$ is a real submanifold of $\mathbb{R}^{2 n}$ of codimension 2 . These singularities are also studied in [24, 23, where it is proved that if the permutation $\sigma$ is the identity, then the function $f$ is smoothly equivalent to a Pham-Brieskorn singularity. Hence in [26] these singularities are called twisted Pham-Brieskorn singularities.

Now we study the transversality between $\mathcal{F}$ and $M$ in special cases.

Case i) Let $n=2$ and $F(\mathbf{z})=\left(\lambda_{1} z_{1}^{a_{1}}, \lambda_{2} z_{2}^{a_{2}}\right)$. Then

$$
M=\left\{\left(z_{1}, z_{2}\right) \in \mathbb{C}^{2} \mid h(\mathbf{z}):=\lambda_{1} z_{1}^{a_{1}} \bar{z}_{1}+\lambda_{2} z_{2}^{a_{2}} \bar{z}_{2}=0\right\},
$$

so $M$ is defined as the zero set of the real functions $\psi(\mathbf{z}):=\operatorname{Re}(h(\mathbf{z}))$ and $\phi(\mathbf{z}):=\operatorname{Im}(h(\mathbf{z}))$. Notice that a vector $v(\mathbf{z})$ belongs to $T_{\mathbf{z}} M$ if and only if $v(\mathbf{z})$ is orthogonal to the gradient of both $\psi(\mathbf{z})$ and $\phi(\mathbf{z})$. On the other hand $\{F(\mathbf{z}), i F(\mathbf{z})\}$ is a basis of the tangent space of the leaf of $\mathcal{F}$ containing $\mathbf{z}$.

Set $\bar{\nabla} \psi(\mathbf{z}):=\left(\frac{\partial \psi}{\partial \bar{z}_{1}}, \ldots, \frac{\partial \psi}{\partial \bar{z}_{n}}\right)$ and consider the gradient vector field of $\psi(\mathbf{z})$. One has:

$$
2\langle F(\mathbf{z}), \bar{\nabla} \psi(\mathbf{z})\rangle_{\mathbb{C}}=\langle F(\mathbf{z}), \operatorname{grad} \psi(\mathbf{z})\rangle_{\mathbb{R}}-i\langle i F(\mathbf{z}), \operatorname{grad} \psi(\mathbf{z})\rangle_{\mathbb{R}} .
$$


Let us set:

$$
A=\left|\lambda_{1}\right|^{2}\left|z_{1}\right|^{2 a_{1}}+\left|\lambda_{2}\right|^{2}\left|z_{2}\right|^{2 a_{2}} \quad, \quad B=a_{1} \lambda_{1}^{2} z_{1}^{2 a_{1}-1} \bar{z}_{1}+a_{2} \lambda_{2}^{2} z_{2}^{2 a_{2}-1} \bar{z}_{2} .
$$

Then, we have:

$$
\begin{gathered}
\langle F(\mathbf{z}), \operatorname{grad} \psi\rangle_{\mathbb{R}}=2 A+2 \operatorname{Re} B \quad, \quad\langle i F(\mathbf{z}), \operatorname{grad} \psi\rangle_{\mathbb{R}}=2 \operatorname{Im} B, \\
\langle F(\mathbf{z}), \operatorname{grad} \phi\rangle_{\mathbb{R}}=-2 \operatorname{Im} B \quad \text { and } \quad\langle i F(\mathbf{z}), \operatorname{grad} \phi\rangle_{\mathbb{R}}=-2 A+2 \operatorname{Re} B .
\end{gathered}
$$

Let $\theta_{k}$ and $\theta_{\lambda_{k}}$ be the arguments of $z_{k}$ and $\lambda_{k}$ respectively. The equations for $M$ can be written as:

$$
\left|\lambda_{1}\right|\left|z_{1}\right|^{a_{1}+1}=\left|\lambda_{2}\right|\left|z_{2}\right|^{a_{2}+1} \quad \text { and } \quad e^{i \theta_{\lambda_{1}}} e^{i\left(a_{1}-1\right) \theta_{1}}=-e^{i \theta_{\lambda_{2}}} e^{i\left(a_{2}-1\right) \theta_{2}} .
$$

Then:

$$
\begin{aligned}
B & =a_{1}\left|\lambda_{1}\right|^{2}\left|z_{1}\right|^{2 a_{1}} e^{i 2 \theta_{\lambda_{1}}} e^{i\left(2 a_{1}-2\right) \theta_{1}}+a_{2}\left|\lambda_{2}\right|^{2}\left|z_{2}\right|^{2 a_{2}} e^{i 2 \theta_{\lambda_{2}}} e^{i\left(2 a_{2}-2\right) \theta_{2}} \\
& =\left(a_{1}\left|\lambda_{1}\right|^{2}\left|z_{1}\right|^{2 a_{1}}+a_{2}\left|\lambda_{2}\right|^{2}\left|z_{2}\right|^{2 a_{2}}\right) e^{i 2 \theta_{\lambda_{2}}} e^{i\left(2 a_{2}-2\right) \theta_{2}} .
\end{aligned}
$$

Assume now that $F(\mathbf{z})$ is orthogonal to $\operatorname{grad} \phi$. Then

$$
B= \pm\left(a_{1}\left|\lambda_{1}\right|^{2}\left|z_{1}\right|^{2 a_{1}}+a_{2}\left|\lambda_{2}\right|^{2}\left|z_{2}\right|^{2 a_{2}}\right) .
$$

Hence

$$
\langle F(\mathbf{z}), \operatorname{grad} \psi\rangle_{\mathbb{R}}=2\left(1 \pm a_{1}\right)\left|\lambda_{1}\right|^{2}\left|z_{1}\right|^{2 a_{1}}+2\left(1 \pm a_{2}\right)\left|\lambda_{2}\right|^{2}\left|z_{2}\right|^{2 a_{2}} .
$$

Thus $F(\mathbf{z})$ is orthogonal to $\operatorname{grad} \psi$ only at the origin. If we develop the equations for $i F(\mathbf{z})$ the results are similar. We thus get that $\mathcal{F}$ is transversal to $M$ in every point away from the origin.

Case ii) Now let $F(\mathbf{z})=\left(\lambda_{1} z_{2}^{a_{2}}, \lambda_{2} z_{1}^{a_{1}}\right)$. The polar variety is defined by:

$$
\lambda_{1} z_{2}^{a_{2}} \bar{z}_{1}+\lambda_{2} z_{1}^{a_{1}} \bar{z}_{2}=0 .
$$

Arguing as above we get that if we assume $F(\mathbf{z})$ is orthogonal to $\operatorname{grad} \phi$, then:

$$
\langle F(\mathbf{z}), \operatorname{grad} \psi\rangle_{\mathbb{R}}=\left(1 \pm a_{1}\right)\left|\lambda_{1}\right|^{2}\left|z_{2}\right|^{2 a_{2}}+\left(1 \pm a_{2}\right)\left|\lambda_{2}\right|^{2}\left|z_{1}\right|^{2 a_{1}} .
$$

This product is zero only if $\mathbf{z}=\mathbf{0}$. Similar computations work also for $i F(\mathbf{z})$. We get that $\mathcal{F}$ and $M$ are transversal away from the origin.

Case iii) Now consider the following vector field in $\mathbb{C}^{4}$ :

$$
F(\mathbf{z})=\left(\lambda_{1} z_{2}^{2}, \lambda_{2} z_{3}^{2}, \lambda_{3} z_{4}^{2}, \lambda_{4} z_{1}^{2}\right) .
$$

The equation of the polar variety is:

$$
h(\mathbf{z}):=\lambda_{1} z_{2}^{2} \bar{z}_{1}+\lambda_{2} z_{3}^{2} \bar{z}_{2}+\lambda_{3} z_{4}^{2} \bar{z}_{3}+\lambda_{4} z_{1}^{2} \bar{z}_{4}=0 .
$$


We claim that for appropriate $\lambda_{i}$, the foliation of $F$ is not transversal to $M^{*}$ at points of the form $\left(z_{1}, z_{2}, z_{3}, 0\right)$. To see this, assume that $F(\mathbf{z})$ is orthogonal to $\operatorname{grad} \psi$ and $\operatorname{grad} \phi$ at one of these points. We get:

$$
\begin{aligned}
0=\frac{1}{2}\langle F(\mathbf{z}), \operatorname{grad} \psi\rangle_{\mathbb{R}} & =\left|\lambda_{1}\right|^{2}\left|z_{2}\right|^{4}+\left|\lambda_{2}\right|^{2}\left|z_{3}\right|^{4}+\left|\lambda_{4}\right|^{2}\left|z_{1}\right|^{4} \pm 2\left|\lambda_{1}\right|^{2}\left|z_{2}\right|^{2}\left|z_{1}\right|^{2} \\
& =\left|\lambda_{1}\right|^{2}\left|z_{2}\right|^{4}+\left|\lambda_{4}\right|^{2}\left|z_{1}\right|^{4}+(1 \pm 2)\left|\lambda_{1}\right|^{2}\left|z_{2}\right|^{2}\left|z_{1}\right|^{2} .
\end{aligned}
$$

Notice that there are two cases to consider, depending to the \pm sign on the last term in this equation. It is clear that with the + sign this equation has no non-trivial solutions. Yet, it is an exercise to show that the equation with the - sign has non-trivial solutions whenever $\left|\lambda_{1}\right|^{2}-4\left|\lambda_{4}\right|^{2}>0$. Therefore in these cases $\mathcal{F}$ is not transversal to $M^{*}$ at points of the form $\left(z_{1}, z_{2}, z_{3}, 0\right)$.

\section{Contact-analytic foliations}

From now on we assume the Morse function $g$ is analytic on $U$.

Definition 3.1. The foliation $\mathcal{F}$ is contact-analytic at $\mathbf{0}$ with respect to $g$ if the germ at $\mathbf{0}$ of the space $M=M(\mathcal{F}, g)$ is real analytic.

Examples 3.2. i) If $\mathcal{F}$ is 1 -dimensional, then it is defined by a holomorphic vector field $F$, and for the usual round metric $M$ is defined by the analytic equations $\langle F(\mathbf{z}), \mathbf{z}\rangle=0$. Thus it is contact-analytic for this metric.

ii) If $\mathcal{F}$ is given by a holomorphic action of some complex Lie group of dimension $d$ with a fixed point at $\mathbf{0}$ and trivial isotropy away from $\mathbf{0}$, then $M$ is contact-analytic for this metric.

iii) If $\mathcal{F}$ has codimension 1 and $n>2$, then by Malgrange's theorem [18] it has a first integral. So we can assume it is defined by a holomorphic map $f$. The tangent space of $\mathcal{F}$ at each point $\mathbf{z}$ is the kernel of $d f$. We will see in Section 6 that $\mathcal{F}$ is contact-analytic for the usual metric.

If the germ of $M$ at $\mathbf{0}$ is real analytic, then it has finitely many irreducible components. By Hironaka's work in [11, we can always equip a small ball around $\mathbf{0} \in \mathbb{C}^{n}$ with a Whitney stratification compatible with $M$, and we can further assume that $\{\mathbf{0}\}$ itself is a stratum and every other stratum of $M^{*}$ has $\{\mathbf{0}\}$ in its closure. Then Verdier's Bertini-Sard theorem 28 implies that sufficiently near the origin, $g$ has no critical points on $M^{*}=M \backslash\{\mathbf{0}\}$ in the stratified sense (compare with [21]). Then one gets that every stratum of $M^{*}$ meets transversally each sufficiently small sphere around $\{\mathbf{0}\}$, and each irreducible component of $M$ at $\mathbf{0}$ is locally a cone over its intersection with a sphere small enough around $\mathbf{0}$ (local conical structure of analytic sets).

If we assume that $\mathcal{F}$ carries the Morse structure of $g$, then $M^{*}$ is non-singular and we can assume that, after removing $\mathbf{0}$, each irreducible component of $M$ at $\mathbf{0}$ becomes a Whitney stratum. In this case one has that the gradient flow $\mathcal{G}_{M^{*}}$ of $\left.g\right|_{M^{*}}$ is transversal to all the $g$-spheres, its orbits accumulate at $\mathbf{0}$ and it equips $M$ with the structure of a cone over $M_{\varepsilon}:=M \cap \mathbb{S}_{\varepsilon}$ with vertex at $\mathbf{0}$. 
Theorem 3.3. Let $\mathcal{F}$ be contact-analytic for the Morse function $g$ and assume it carries the Morse structure of $g$. Let $M_{1}, \ldots, M_{r}$ be the irreducible components of the polar variety $M$. Then:

i) Each $M_{i}^{*}:=M_{i} \backslash\{\mathbf{0}\}$ is a real submanifold of $\mathbb{C}^{n}$ of codimension $2 d$, transversal to $\mathcal{F}$ everywhere and transversal to all the $g$-spheres;

ii) The $M_{i}$ 's are pairwise disjoint away from $\mathbf{0}$ and each $M_{i}^{*}$ consists of points with the same Morse index.

Proof. Item (ii) is the only statement that needs a proof, the other statement is an immediate consequence of Theorem 2.1. For proving (ii), supose we have points $p_{1}$ and $p_{2}$ in some $M_{i}$ with different Morse index, and consider an arc $\alpha$ in $M_{i}$ joining these points. Then necessarily exits a point $q$ in this arc which is a degenerate contact, because Morse singularities are stable and we cannot have a continuous family of Morse singularities with distinct Morse indices.

\section{Morse theory and the topology of the leaves}

Recall from Section 1 that given a leaf $\mathcal{L}$ of $\mathcal{F}$, we denote by $g_{\mathcal{L}}$ the restriction of $g$ to $\mathcal{L}$. Lemma 1.2 says that the critical points of $g_{\mathcal{L}}$ are the points where $\mathcal{L}$ is tangent to the level surfaces of $g$.

The $\alpha$-limit of a point $\mathbf{z} \in \mathcal{U}$ under the flow $\mathcal{G}_{\mathcal{F}}$, is the set $\alpha(\mathbf{z})$ consisting of all points $\mathbf{y} \in \mathcal{U}$ for which we can find a sequence $t_{n} \in \mathbb{R}$, such that $\lim _{t_{n} \rightarrow-\infty} \mathcal{G}_{\mathcal{L}}\left(t_{n}, \mathbf{z}\right)=\mathbf{y}$. The $\alpha$-limit of a saturated set is the union of the $\alpha$-limits of points in that set.

The proof of the following two lemmas is an exercise, and it is exactly as the proof of propositions 3.1 and 3.2 in 9 for 1-dimensional foliations. Notice that in the case of the usual metric, the gradient flow we consider here is the opposite of the radial flow envisaged in that article, so we consider $\alpha$-limits instead of $\omega$-limits.

Lemma 4.1. If $M$ carries a Morse structure, then the $\alpha$-limit of $\mathcal{G}_{\mathcal{F}}$ is the polar variety $M$.

Lemma 4.2. Let $\widehat{M}$ be the saturated of $M$ by $\mathcal{F}$, i.e., the union of all leaves that meet $M$, and let $K:=\mathcal{U}_{\varepsilon} \backslash \widehat{M}$. Then the $\alpha$-limit of $K$ by the flow $\mathcal{G}_{\mathcal{F}}$ is the origin $\mathbf{0}$, and the $\alpha$-limit of each $\mathbf{z} \in \widehat{M}$ is the origin or the point where the corresponding flow line meets $M$.

We now have:

Theorem 4.3. Assume $\mathcal{F}$ is contact-analytic for $g$ and carries its Morse structure. We now restrict $\mathcal{F}$ to a small closed ball $\mathbb{B}_{\varepsilon}$ centred at $\mathbf{0} \in \mathbb{C}^{n}$. Let $M$ be the intersection with $\mathbb{B}_{\varepsilon}$ of the corresponding polar variety in $\mathcal{U}$, let $\widehat{M}$ be the saturated of $M$ by $\mathcal{F}$ in $\mathbb{B}_{\varepsilon}$ and set $K:=\mathbb{B}_{\varepsilon} \backslash \widehat{M}$. Then:

1. The gradient flow endows $K$ with the structure of a foliated cone with deleted vertex at $\mathbf{0}$. In fact, each leaf $\mathcal{L} \subset K$ is transversal to all the 
$g$-spheres around $\mathbf{0}$, it is diffeomorphic to the product $\left(\mathcal{L} \cap \mathbb{S}_{\varepsilon}\right) \times \mathbb{R}$ and it has the origin in its closure.

2. If $\mathcal{L}$ is compact, then its Euler-Poincaré characteristic $\chi(\mathcal{L})$ equals the number of intersection points in $\mathcal{L} \cap M$, counted with sign. The sign is negative when the corresponding Morse index is odd, and positive otherwise.

Proof. Item (1) follows immediately from the two lemmas above. Item (2) follows from the considerations above and the theorem of Poincaré-Hopf for manifolds with boundary, since the vector field corresponding to the gradient flow on the leaves is transversal to the boundary. Hence the Euler-Poincaré characteristic $\chi(\mathcal{L})$ equals the total Poincaré-Hopf index of this flow on the leaf. At each singularity of the flow, the local Poincaré-Hopf index is determined by the corresponding Morse index: it is 1 when the Morse index is even (or 0), and it is -1 when the Morse index is odd.

Remark 4.4. Notice that if a leaf $\mathcal{L} \subset \widehat{M}$ is compact, then not only its EulerPoincaré characteristic is determined as above, but actually classical Morse theory tells us that its whole topology is determined by its intersection with the boundary sphere and the Morse indices at the points where $\mathcal{L}$ meets $M$. To some extent, this comments apply also for non-compact leaves, but here the situation can be far more complicated.

\section{The foliation on the spheres}

Notice that given the germ $(\mathcal{F}, \mathbf{0})$ and a small $g$-sphere $\mathbb{S}_{\varepsilon}$, the intersection of the leaves of $\mathcal{F}$ with $\mathbb{S}_{\varepsilon}$ defines a real analytic foliation $\mathcal{F}_{\varepsilon, g}$ on the sphere, which is singular at $M_{\varepsilon}(\mathcal{F}, g):=\mathbb{S}_{\varepsilon} \cap M(\mathcal{F}, g)$. Away from $M_{\varepsilon}(\mathcal{F}, g)$ the leaves of $\mathcal{F}_{\varepsilon, g}$ have real dimension $2 d-1$.

Let us assume $\mathcal{F}$ is contact-analytic and carries the Morse structure of $g$. Let $M_{1}, \ldots, M_{r}$ be the irreducible components of the germ $(M, \mathbf{0})$. For $\varepsilon>0$ sufficiently small, each of these components meets $\mathbb{S}_{\varepsilon}$ transversally in a smooth submanifold of the sphere, called the link $L_{i, \varepsilon}$ of the corresponding singularity. It is well known, by work of Milnor and others (see for instance [?, Theorem 1.15]) that there exists a homeomorphism of the form:

$$
\left(\mathbb{B}_{\varepsilon}, \mathbb{B}_{\varepsilon} \cap\left(\cup M_{i}\right)\right) \longrightarrow \operatorname{Cone}\left(\mathbb{S}_{\varepsilon}, \cup L_{i, \varepsilon}\right) .
$$

On the other hand, for all $\varepsilon>\varepsilon^{\prime}>0$ small enough we have a diffeomorphism sending $\mathbb{S}_{\varepsilon}$ into $\mathbb{S}_{\varepsilon}^{\prime}$ and the singular set of $\mathcal{F}_{\varepsilon, g}$ into the corresponding one for $\mathcal{F}_{\varepsilon^{\prime}, g}$, i.e., $\left(\mathbb{S}_{\varepsilon}, \bigcup L_{i, \varepsilon}\right) \cong\left(\mathbb{S}_{\varepsilon}^{\prime}, \bigcup L_{i, \varepsilon^{\prime}}\right)$. Thus, it is natural to ask whether the corresponding foliations are topologically conjugate. That is:

Question 1. Is there a homeomorphism (or a diffeomorphism) from $\mathbb{S}_{\varepsilon}$ into $\mathbb{S}_{\varepsilon}^{\prime}$ carrying the leaves of $\mathcal{F}_{\varepsilon, g}$ into those of $\mathcal{F}_{\varepsilon^{\prime}, g}$ ? In other words, are these foliations equivalent, either topologically or differentiably? 
The answer in general seems to be negative, though it is positive in some settings, as shown by the theorem below. This result is an extension of the equivalent theorem in [9] for 1-dimensional foliations.

Theorem 5.1. If the Morse index of each point in $M^{*}$ is 0 , then for all $\varepsilon>\varepsilon^{\prime}>$ 0 small enough, the induced foliations on the spheres are smoothly equivalent. That is, there is a diffeomorphism

$$
\left(\mathbb{S}_{\varepsilon}, \mathcal{F}_{\varepsilon}, M_{\varepsilon}\right) \stackrel{\cong}{\longrightarrow}\left(\mathbb{S}_{\varepsilon^{\prime}}, \mathcal{F}_{\varepsilon^{\prime}}, M_{\varepsilon^{\prime}}\right),
$$

taking leaves of $\mathcal{F}_{\varepsilon}$ into leaves of $\mathcal{F}_{\varepsilon^{\prime}}$, where $M_{\varepsilon}$ and $M_{\varepsilon^{\prime}}$ are the singular sets of $\mathcal{F}_{\varepsilon}$ and $\mathcal{F}_{\varepsilon^{\prime}}$, respectively.

Proof. All points in $M^{*}$ have Morse index 0 , so they are points of local minimal $g$-distance to $\mathbf{0}$ in their leaves. Hence every leaf of $\mathcal{F}_{\varepsilon}$ near $M$ is compact and diffeomorphic to a sphere of dimension $2 d-1$. Let $V_{\varepsilon}$ be a tubular neighbourhood of $M_{\varepsilon}$ in $\mathbb{S}_{\varepsilon}$ such that its boundary $\partial V_{\varepsilon}$ is formed by such leaves.

Let $\mathcal{G}_{M^{*}}$ be the gradient flow of $\left.g\right|_{M^{*}}$, as in Section 3 Its orbits accumulate at $\mathbf{0}$ and they are transversal to all sufficiently small $g$-spheres. This gives a diffeomorphism $M_{\varepsilon} \rightarrow M_{\varepsilon}^{\prime}$ as in [21], which is our starting point for comparing the foliation $\mathcal{F}_{\varepsilon}$ with the foliation $\mathcal{F}_{\varepsilon^{\prime}}$ for $\varepsilon>\varepsilon^{\prime}>0$. The claim (to be proved) is that these foliations are topologically, and actually smoothly, equivalent. By compactness, we can assume that $\varepsilon^{\prime}$ is close enough to $\varepsilon$ so that every leaf in $\partial V_{\varepsilon}$ meets $\mathbb{S}_{\varepsilon^{\prime}}$ transversally, for otherwise we can take a finite number of spheres with $\varepsilon=\varepsilon_{0}>\varepsilon_{1}>\cdots>\varepsilon_{r}=\varepsilon^{\prime}$ and apply the following arguments step by step.

Consider the gradient flow $\mathcal{G}_{M^{*}}$, and for each $\mathbf{z} \in M_{\varepsilon}$, let $\gamma_{t}(\mathbf{z})$ be the flow line of $\mathcal{G}_{M^{*}}$ passing through $\mathbf{z}$. Notice that $\gamma_{t}(\mathbf{z})$ intersects every sphere $\mathbb{S}_{\varepsilon_{t}}$, $\varepsilon \geq \varepsilon_{t} \geq \varepsilon^{\prime}$, in a single point. We call $\mathbf{y}(\mathbf{z})$ the corresponding point in $\mathbb{S}_{\varepsilon^{\prime}}$. Each point in $\gamma_{t}(\mathbf{z})$ between the points $\mathbf{z}$ and $\mathbf{y}(\mathbf{z})$ belongs to a leaf $\mathcal{L}$ in $\mathcal{F}$ such that the intersection $\mathcal{L} \cap S_{\varepsilon}$ is a sphere contained in the interior of the tubular neighbourhood $V_{\varepsilon}$. Also, each leaf $\mathcal{L}_{\partial}$ in $\partial V_{\varepsilon}$ corresponds to a point $\mathbf{p}\left(\mathcal{L}_{\partial}\right)$ in $M$ whose $g$-distance to $\mathbf{0}$ is strictly less than $\varepsilon^{\prime}$, which is the point of contact of $\mathcal{L}_{\partial}$ with a $g$-sphere.

For each leaf $\mathcal{L}_{\partial}$ in $\partial V_{\varepsilon}$, let $\mathbf{p}\left(\mathcal{L}_{\partial}\right)$ be its point of contact in $M$, as above. Let $\gamma_{t}\left(\mathbf{p}\left(\mathcal{L}_{\partial}\right)\right)$ be the flow line through $\mathbf{p}\left(\mathcal{L}_{\partial}\right)$, let $\mathbf{z}\left(\mathcal{L}_{\partial}\right)$ be the point where this arc meets $\mathbb{S}_{\varepsilon}$ and $\mathbf{y}\left(\mathcal{L}_{\partial}\right)$ the point where $\gamma_{t}\left(\mathbf{p}\left(\mathcal{L}_{\partial}\right)\right)$ meets $\mathbb{S}_{\mathcal{\varepsilon}^{\prime}}$. The points in the arc $\left[\mathbf{p}\left(\mathcal{L}_{\partial}\right), \mathbf{z}\left(\mathcal{L}_{\partial}\right)\right)$ in the curve $\gamma_{t}\left(\mathbf{p}\left(\mathcal{L}_{\partial}\right)\right)$ determine a 1-parameter family of $(2 d-1)$ spheres in $\mathbb{S}_{\varepsilon}$, nested around the point $\mathbf{z}\left(\mathcal{L}_{\partial}\right)$, forming a disc $\mathbb{D}_{\varepsilon, \mathbf{z}}^{\mathcal{N}}$ of dimension $2 d$, transversal to $M^{*}$ at $\mathbf{z}\left(\mathcal{L}_{\partial}\right)$. Notice that the interval $\left[\mathbf{p}\left(\mathcal{L}_{\partial}\right), \mathbf{z}\left(\mathcal{L}_{\partial}\right)\right)$ is taken to be open on the right because the end-point corresponds to the centre of the disc. Doing so for all points in $M_{\varepsilon}$ we get back the neighbourhood $V_{\varepsilon}$, naturally identified with the normal bundle of $M_{\varepsilon}$ in the sphere, and each normal fibre $\mathbb{D}_{\varepsilon, \mathbf{z}}^{\mathcal{N}}$ is foliated by concentric spheres which are leaves of $\mathcal{F}_{\varepsilon}$.

Similarly, the points in the $\operatorname{arc}\left[\mathbf{p}\left(\mathcal{L}_{\partial}\right), \mathbf{y}\left(\mathcal{L}_{\partial}\right)\right)$ in the curve $\gamma_{t}\left(\mathbf{p}\left(\mathcal{L}_{\partial}\right)\right)$ determine a 1-parameter family of $(2 d-1)$ spheres in $\mathbb{S}_{\mathcal{E}^{\prime}}$, nestled around the point 
$\mathbf{y}\left(\mathcal{L}_{\partial}\right)$, forming a disc $\mathbb{D}_{\varepsilon^{\prime}, \mathbf{y}}^{\mathcal{N}}$ of dimension $2 d$, transversal to $M^{*}$ at $\mathbf{y}\left(\mathcal{L}_{\partial}\right)$. Doing so for all points in $M_{\varepsilon^{\prime}}$ we get a tubular neighbourhood $V_{\varepsilon^{\prime}}$ of $M_{\varepsilon^{\prime}}$ in $\mathbb{S}_{\varepsilon^{\prime}}$.

Let $\mathcal{C}_{\varepsilon, \varepsilon^{\prime}}$ be the cylinder bounded by the spheres $\mathbb{S}_{\varepsilon}$ and $\mathbb{S}_{\varepsilon^{\prime}}$. This cylinder is a union of two compact foliated sets $\mathcal{A}_{\varepsilon, \varepsilon^{\prime}}, \mathcal{V}_{\varepsilon, \varepsilon^{\prime}}$ obtained as follows: If $\stackrel{\circ}{V}_{\varepsilon}$ is the interior of $V_{\varepsilon}$, then $\mathcal{A}_{\varepsilon, \varepsilon^{\prime}}$ is the subset of $\mathcal{C}_{\varepsilon, \varepsilon^{\prime}}$ obtained by saturating $\mathbb{S}_{\varepsilon} \backslash \stackrel{\circ}{V}_{\varepsilon}$ by $\mathcal{F}$. This is a cylinder foliated by the leaves of $\mathcal{F}$ intersected with $\mathcal{C}_{\varepsilon, \varepsilon^{\prime}}$. Each leaf in $\mathcal{A}_{\varepsilon, \varepsilon^{\prime}}$ is transversal to all the $g$-spheres of radius $r$ with $\varepsilon \geq r \geq \varepsilon^{\prime}$. The set $\mathcal{V}_{\varepsilon, \varepsilon^{\prime}}$ is the compact neighbourhood of $M \cap \mathcal{C}_{\varepsilon, \varepsilon^{\prime}}$ in $\mathcal{C}_{\varepsilon, \varepsilon^{\prime}}$, obtained by saturating $V_{\varepsilon}$ by $\mathcal{F}$. Notice that the intersection of these two sets $\mathcal{C}_{\varepsilon, \varepsilon^{\prime}}, \mathcal{V}_{\varepsilon, \varepsilon^{\prime}}$ is exactly the subset obtained by saturating $\partial V_{\varepsilon}$.

Let us prove now that the foliations $\mathcal{F}_{\varepsilon}$ and $\mathcal{F}_{\varepsilon^{\prime}}$ are smoothly equivalent. We do it in pieces, first on $\mathcal{A}_{\varepsilon, \varepsilon^{\prime}}$. Let $\mathcal{G}_{\mathcal{F}}$ be the gradient flow on the leaves in $\mathcal{A}_{\varepsilon, \varepsilon^{\prime}}$, defined in Section 4. This is a smooth flow on all of $\mathcal{A}_{\varepsilon, \varepsilon^{\prime}}$, with no singularities, and its flow lines are transversal to all the spheres. This determines a diffeomorphism

$$
H:\left(\mathbb{S}_{\varepsilon} \backslash \stackrel{\circ}{V} \varepsilon\right) \longrightarrow\left(\mathbb{S}_{\varepsilon^{\prime}} \backslash \stackrel{\circ}{V_{\varepsilon^{\prime}}}\right)
$$

that carries leaves of $\mathcal{F}_{\varepsilon}$ into leaves of $\mathcal{F}_{\varepsilon^{\prime}}$.

Notice that $H$ defines a diffeomorphism from $\partial V_{\varepsilon}$ into $\partial V_{\varepsilon^{\prime}}$. We now extend $H$ to the interior of these neighbourhoods. Recall that a diffeomorphism is already given between $M_{\varepsilon}$ and $M_{\varepsilon^{\prime}}$ and it is defined by the gradient flow $\mathcal{G}_{M^{*}}$. For the moment, denote this map by $h$. This is compatible with $H$ in the sense that if $V_{\varepsilon}$ and $V_{\varepsilon^{\prime}}$ are identified with the unit disc normal bundles of $M_{\varepsilon}$ and $M_{\varepsilon^{\prime}}$ in the corresponding spheres, then for each $\mathbf{z} \in M_{\varepsilon}, H$ is a bundle map between the corresponding sphere bundles. That is, for each $\mathbf{z} \in M_{\varepsilon}, H$ carries the boundary of the normal disc $\mathbb{D}_{\varepsilon, \mathbf{z}}^{\mathcal{N}}$ into the boundary of the normal disc $\mathbb{D}_{\varepsilon^{\prime}, h(\mathbf{z})}^{\mathcal{N}}$. Thus we denote $h$ also by $H$.

It remains to extend $H$ to the interior of the normal discs $\mathbb{D}_{\varepsilon, \mathbf{z}}^{\mathcal{N}}, \mathbb{D}_{\varepsilon^{\prime}, H(\mathbf{z})}^{\mathcal{N}}$, and we have already defined it on $M^{*}$ in a compatible way. Recall that each such disc is foliated by spheres and these are parameterized by an $\operatorname{arc}\left[\mathbf{p}\left(\mathcal{L}_{\partial}\right), \mathbf{z}\left(\mathcal{L}_{\partial}\right)\right)$, contained in an integral line of the gradient flow on $M^{*}$, where $\mathcal{L}_{\partial}$ is the boundary of the disc $\mathbb{D}_{\varepsilon, \mathbf{z}}^{\mathcal{N}}, \mathbf{p}\left(\mathcal{L}_{\partial}\right)$ is the point where the leaf of $\mathcal{F}$ that determines $\mathcal{L}_{\partial}$ meets $M$, and $\mathbf{z}=\mathbf{z}\left(\mathcal{L}_{\partial}\right)$ is the point in $M_{\varepsilon}$ whose normal fibre is determined by $\mathcal{L}_{\partial}$. Similarly, the disc $\mathbb{D}_{\varepsilon^{\prime}, H(\mathbf{z})}^{\mathcal{N}}$ is parameterized by the $\operatorname{arc}\left[\mathbf{p}\left(\mathcal{L}_{\partial}\right), H(\mathbf{z})\right) \subset\left[\mathbf{p}\left(\mathcal{L}_{\partial}\right), \mathbf{z}\right)$.

Choose a smooth family of diffeomorphisms, parameterized by $M_{\varepsilon}$, that carry each interval $\left[\mathbf{p}\left(\mathcal{L}_{\partial}\right), \mathbf{z}\right)$ into the corresponding interval $\left[\mathbf{p}\left(\mathcal{L}_{\partial}\right), H(\mathbf{z})\right)$. This determines which sphere in each $\mathbb{D}_{\varepsilon, \mathbf{z}}^{\mathcal{N}}$ goes to which sphere in $\mathbb{D}_{\varepsilon^{\prime}, H(\mathbf{z})}^{\mathcal{N}}$. The rest is now an exercise, using that the diffeomorphism is already given on the boundary of each disc. For this we construct integrable vector fields on $V_{\varepsilon}$ and $V_{\varepsilon^{\prime}}$, which are tangent to each disc $\mathbb{D}_{\varepsilon, \mathbf{z}}^{\mathcal{N}}, \mathbb{D}_{\varepsilon^{\prime}, H(\mathbf{z})}^{\mathcal{N}}$, transversal to each leaf (sphere) in these discs, and singular at the center of the corresponding disc. These vector fields can be regarded as being families parameterized by the points in $M_{\varepsilon}$ and $M_{\varepsilon^{\prime}}$ respectively, of radial vector fields on each normal disc $\mathbb{D}_{\varepsilon, \mathbf{z}}^{\mathcal{N}}, \mathbb{D}_{\varepsilon^{\prime}, H(\mathbf{z})}^{\mathcal{N}}$. These vector fields give us a natural way for extending the given 
diffeomorphisms

$$
\partial \mathbb{D}_{\varepsilon, \mathbf{z}}^{\mathcal{N}} \rightarrow \partial \mathbb{D}_{\varepsilon^{\prime}, H(\mathbf{z})}^{\mathcal{N}}
$$

to the interior of the discs, carrying leaves of $\mathcal{F}_{e}$ into leaves of $\mathcal{F}_{\varepsilon^{\prime}}$.

We thus get an extension of $H$ to a diffeomorphism $\left(\mathbb{S}_{\varepsilon}, M_{\varepsilon}\right) \rightarrow\left(\mathbb{S}_{\varepsilon^{\prime}}, M_{\varepsilon}^{\prime}\right)$, taking leaves of $\mathcal{F}_{\varepsilon}$ into leaves of $\mathcal{F}_{\varepsilon^{\prime}}$,

\section{Codimension one foliations}

We now restrict the discussion to codimension one foliations. We assume further that $\mathcal{F}$ has a first integral near $\mathbf{0}$, so there exists a holomorphic map-germ $\left(\mathbb{C}^{n}, \mathbf{0}\right) \stackrel{f}{\rightarrow}(\mathbb{C}, 0)$ such that near $\mathbf{0}$, the leaves of $\mathcal{F}$ are the fibers of $f$. By Malgrange's theorem in 18, this hypothesis is a restriction only when $n=2$.

Our first task is to find a suitable set of generators for the tangent bundle $T \mathcal{F}$ of $\mathcal{F}$ away from $\mathbf{0}$ (to include the origin we must speak of the tangent sheaf). For instance, if $n=2$, then the holomorphic vector field $F=\left(\frac{\partial f}{\partial z_{2}},-\frac{\partial f}{\partial z_{1}}\right)$ satisfies $d f(F(\mathbf{z}))=0$ for all $\mathbf{z} \in \mathcal{U}$. Since $T \mathcal{F}$ is 1 -dimensional, we have that $F$ determines a basis for $T \mathcal{F}$ at all points in $\mathcal{U} \backslash\{\mathbf{0}\}$.

For $n>2$ one has that the tangent sheaf of $\mathcal{F}$ may not be locally free. Thence, generally speaking, we need more than $n-1$ germs of holomorphic vectors fields to generate $T \mathcal{F}$. The proof of the following lemma is an exercise:

Lemma 6.1. Consider the vector fields

$$
v_{j, k}(\mathbf{z}):=\left(0, \ldots, 0, \frac{\partial f}{\partial z_{k}}(\mathbf{z}), 0, \ldots, 0,-\frac{\partial f}{\partial z_{j}}(\mathbf{z}), 0, \ldots, 0\right),
$$

where the entries $\frac{\partial f}{\partial z_{k}}(\mathbf{z})$ and $-\frac{\partial f}{\partial z_{j}}(\mathbf{z})$ are in the $j$ th and $k$ th position respectively. Then $\left\{v_{j, k}\right\}_{1 \leq j<k \leq n}$ is a set of generators of each tangent space $T_{\mathbf{z}} \mathcal{L}_{\mathbf{z}}$, for all $\mathbf{z} \in \mathcal{U} \backslash\{\mathbf{0}\}$.

Of course this lemma is giving us too many generators, which can be unsatisfactory in some sense. Yet, these vector fields are so simple and canonical, that they lead to nice expressions for the polar variety of $M$. This will be useful in the sequel.

As before, let $M(\mathcal{F}, g)$ be the polar variety of $\mathcal{F}$ with respect to a Morse function $g$ with Morse index 0 at $\mathbf{0}$. We have:

Proposition 6.2. The variety $M(\mathcal{F}, g)$ is defined by the equations

$$
\frac{\partial g}{\partial z_{k}} \frac{\partial f}{\partial z_{j}}=\frac{\partial g}{\partial z_{j}} \frac{\partial f}{\partial z_{k}}
$$

for all $j, k$ in $\{1, \ldots, n\}$. Hence, if $g$ is analytic then $\mathcal{F}$ is contact-analytic with respect to $g$. In particular, for $Q\left(z_{1}, \ldots, z_{n}\right)=\left|z_{1}\right|^{2}+\ldots+\left|z_{n}\right|^{2}$ we obtain:

$$
M(\mathcal{F}, Q)=\left\{\mathbf{z} \in \mathbb{C}^{n} \mid \bar{z}_{k} \frac{\partial f}{\partial z_{j}}(\mathbf{z})=\bar{z}_{j} \frac{\partial f}{\partial z_{k}}(\mathbf{z}), 1 \leq j<k \leq n\right\} .
$$


Proof. Notice that the last two statements in 6.2 follow from the equations (3), so we only need to show that these equations actually define $M$. Since $f$ has an isolated critical point at $\mathbf{0}$, given a point $\mathbf{z}$ in $\mathbb{C}^{n} \backslash\{\mathbf{0}\}$ one has $\frac{\partial f}{\partial z_{j}}(\mathbf{z}) \neq 0$ for some $j$. Let $\mathcal{L}$ be the leaf of $\mathcal{F}$ containing $\mathbf{z}$. Then the implicit function theorem says that there is a neigborhood $U^{\prime}$ of $\mathbf{z}$ where, on the leaf $\mathcal{L}$, the coordinate function $z_{j}$ depends holomorphically on the other variables. Deriving implicitly the equation $f(\mathbf{z})=c$ we find:

$$
\frac{\partial z_{j}}{\partial z_{k}}=-\frac{\frac{\partial f}{\partial z_{k}}}{\frac{\partial f}{\partial z_{j}}}
$$

at all points in $U^{\prime} \cap \mathcal{L}$. Using this to find the critical points of $g_{\mathcal{L}}$ we obtain the desired equations by a straight-forward computation.

We know from the examples in 1.7 that there are foliations that have degenerate contact points for the function $Q\left(x_{1}, y_{1}, x_{2}, y_{2}\right)=x_{1}^{2}+y_{1}^{2}+x_{2}^{2}+y_{2}^{2}$, as for instance the foliation defined by the polynomial map $f\left(z_{1}, z_{2}\right)=z_{1}^{2}+z_{2}^{2}$. Yet, in these example one can easily destroy the degenerate contacts. For instance:

Examples 6.3. Assume $Q_{\Lambda}\left(x_{1}, y_{1}, x_{2}, y_{2}\right)=a_{1} x_{1}^{2}+b_{1} y_{1}^{2}+a_{2} x_{2}^{2}+b_{2} y_{2}^{2}$ where $a_{1}, b_{1}, a_{2}, b_{2}$ are positive real numbers, and consider the foliation $\mathcal{F}$ defined by the function $f\left(z_{1}, z_{2}\right)=z_{1}^{2}+z_{2}^{2}$. The polar variety is the set defined by

$$
M=\left\{\left(z_{1}, z_{2}\right) \in \mathbb{C}^{2} \mid z_{2}\left[a_{1} \operatorname{Re} z_{1}-i b_{1} \operatorname{Im} z_{1}\right]=z_{1}\left[a_{2} \operatorname{Re} z_{2}-i b_{2} \operatorname{Im} z_{2}\right]\right\} .
$$

If we have $a_{1}>a_{2}$ and $b_{1}>b_{2}$, then the polar variety is formed only by the two coordinate axis, all contacts are non degenerate and each leaf has two saddle points on the $z_{1}$-axe and two minimal points on the $z_{2}$-axe. Analogous statements hold for $a_{1}<a_{2}$ and $b_{1}<b_{2}$.

Notice that in this example the foliation $\mathcal{F}$ is determined by the linear form $d f=2 z_{1} d z_{1}+2 z_{2} d z_{2}$. In [12], T. Ito and B. Scardua prove:

Theorem [Ito-Scardua]. Every linear foliation $\mathcal{F}$ on $\mathbb{C}^{n}$ can be arbitrarily approximated by foliations that carry the Morse structure of $Q$.

Let us consider now homogeneous foliations of degree more than 1 . This means, by Malgrange's theorem, that the foliation $\mathcal{H}_{k}$ is defined by the differential of some homogeneous polynomial of degree more than 2 . The simplest case is:

Theorem 6.4. Let $H_{k}^{n}$ be the Fermat polynomial $\lambda_{1} z_{1}^{k}+\lambda_{2} z_{2}^{k}+\cdots+\lambda_{n} z_{n}^{k}$. If $k>2$, then the foliation $\mathcal{H}_{k}$ defined by the fibers of $H_{k}^{n}$ has a Morse structure compatible with the quadratic form $Q=\left|z_{1}\right|^{2}+\left|z_{2}\right|^{2}+\cdots+\left|z_{n}\right|^{2}$, and the corresponding polar variety consists of a finite number of complex lines through the origin.

That $\mathcal{H}_{k}$ has a Morse structure compatible with the quadratic form $Q$ is an immediate consequence of Theorem 1 and the two propositions below. Notice that the claim that $M$ is a union of complex lines was proved in 12 for linear foliations 
Proposition 6.5. Let $H_{k}^{n}=\lambda_{1} z_{1}^{k}+\lambda_{2} z_{2}^{k}+\cdots+\lambda_{n} z_{n}^{k}$. If $k>2$ then away from $\mathbf{0}$, the polar variety $M=M\left(\mathcal{H}_{k}, Q\right)$ is a differentiable reduced submanifold of $\mathbb{R}^{2 n}$, of dimension 2 .

Proof. For simplicity assume $z_{1} \neq 0$. In this case, the set of vectors

$$
\begin{aligned}
v_{2}= & \left(\lambda_{2} z_{2}^{k-1},-\lambda_{1} z_{1}^{k-1}, 0,0, \ldots, 0\right) \\
v_{3}= & \left(\lambda_{3} z_{3}^{k-1}, 0,-\lambda_{1} z_{1}^{k-1}, 0, \ldots, 0\right) \\
\vdots & \vdots \\
v_{n}= & \left(\lambda_{n} z_{n}^{k-1}, 0,0, \ldots, 0,-\lambda_{1} z_{1}^{k-1}\right)
\end{aligned}
$$

forms a basis of $T_{\mathbf{z}} \mathcal{L}_{\mathbf{z}}$. Then the polar variety is defined by the equations

$$
G_{j}(\mathbf{z}):=\lambda_{1} z_{1}^{k-1} \bar{z}_{j}-\lambda_{j} z_{j}^{k-1} \bar{z}_{1}=0
$$

for all $j=2, \ldots, n$. In other words, $M$ is the zero set of the real equations:

$$
\psi_{j}(z):=2 \operatorname{Re} G_{j}(z) \quad \text { and } \quad \phi_{j}(z):=2 \operatorname{Im} G_{j}(z)
$$

for all $j=2, \ldots, n$. We set $\Phi:=\left(\psi_{2}, \phi_{2}, \ldots, \psi_{n}, \phi_{n}\right)$. Notice one has:

$$
\begin{gathered}
\frac{\partial \psi_{j}}{\partial z_{l}}=\left\{\begin{array}{cc}
\lambda_{1}(k-1) z_{1}^{k-2} \bar{z}_{j}-\bar{\lambda}_{j} \bar{z}_{j}^{k-1} & l=1 \\
-\lambda_{j}(k-1) z_{j}^{k-2} \bar{z}_{1}+\bar{\lambda}_{1} \bar{z}_{1}^{k-1} & l=j \\
0 & l \neq j, 1
\end{array}\right. \\
\frac{\partial \psi_{j}}{\partial \bar{z}_{l}}=\left\{\begin{array}{cc}
\bar{\lambda}_{1}(k-1) \bar{z}_{1}^{k-2} z_{j}-\lambda_{j} z_{j}^{k-1} & l=1 \\
-\bar{\lambda}_{j}(k-1) \bar{z}_{j}^{k-2} z_{1}+\lambda_{1} z_{1}^{k-1} & l=j \\
0 & l \neq j, 1
\end{array}\right. \\
\frac{\partial \phi_{j}}{\partial z_{l}}=\left\{\begin{array}{cc}
i\left[\lambda_{1}(k-1) z_{1}^{k-2} \bar{z}_{j}+\bar{\lambda}_{j} \bar{z}_{j}^{k-1}\right] & l=1 \\
i\left[-\lambda_{j}(k-1) z_{j}^{k-2} \bar{z}_{1}-\bar{\lambda}_{1} \bar{z}_{1}^{k-1}\right] & l=j \\
0 & l \neq j, 1
\end{array}\right. \\
\frac{\partial \phi_{j}}{\partial \bar{z}_{l}}=\left\{\begin{array}{cc}
i\left[-\bar{\lambda}_{1}(k-1) \bar{z}_{1}^{k-2} z_{j}-\lambda_{j} z_{j}^{k-1}\right] & l=1 \\
i\left[\bar{\lambda}_{j}(k-1) \bar{z}_{j}^{k-2} z_{1}+\lambda_{1} z_{1}^{k-1}\right] & l=j \\
0 & l \neq j, 1
\end{array}\right.
\end{gathered}
$$

Set $a_{j}(\mathbf{z}):=\lambda_{1}(k-1) z_{1}^{k-2} \bar{z}_{j}, b_{j}(\mathbf{z}):=\lambda_{j} z_{j}^{k-1}, c_{j}(\mathbf{z}):=\lambda_{j}(k-1) z_{j}^{k-2} \bar{z}_{1}$ and $d(\mathbf{z}):=\lambda_{1} z_{1}^{k-1}$. Then the jacobian matrix $D \Phi$ is (we omit writing the variable $\mathbf{z}$ for simplicity):

$$
\left(\begin{array}{ccccccc}
a_{2}-\bar{b}_{2} & -b_{2}+\bar{a}_{2} & -c_{2}+\bar{d} & d-\bar{c}_{2} & \ldots & 0 & 0 \\
i\left[a_{2}+\bar{b}_{2}\right] & i\left[-b_{2}-\bar{a}_{2}\right] & i\left[-c_{2}-\bar{d}\right] & i\left[d+\bar{c}_{2}\right] & \ldots & 0 & 0 \\
a_{3}-\bar{b}_{3} & -b_{3}+\bar{a}_{3} & 0 & 0 & \ldots & 0 & 0 \\
i\left[a_{3}+\bar{b}_{3}\right] & i\left[-b_{3}-\bar{a}_{3}\right] & 0 & 0 & \ldots & 0 & 0 \\
\vdots & \vdots & \vdots & \vdots & \ddots & \vdots & \vdots \\
a_{n}-\bar{b}_{n} & -b_{n}+\bar{a}_{n} & 0 & 0 & \ldots & -c_{n}+\bar{d} & d-\bar{c}_{n} \\
i\left[a_{n}+\bar{b}_{n}\right] & i\left[-b_{n}-\bar{a}_{n}\right] & 0 & 0 & \ldots & i\left[-c_{n}-\bar{d}\right] & i\left[d+\bar{c}_{n}\right]
\end{array}\right)
$$


To prove that $D \Phi$ has rank $2 n-2$ consider its submatrix $B_{n}$ obtained by eliminating the first two columns of $D \Phi$. Then Proposition 6.5 follows from the following lemma.

Lemma 6.6. Let $B_{n}$ be as above. Then:

$$
\operatorname{det}\left(B_{n}(\mathbf{z})\right)=(2 i)^{n-1} \prod_{j=2}^{n}\left[|d(\mathbf{z})|^{2}-\left|c_{j}(\mathbf{z})\right|^{2}\right]=(2 i)^{n-1} \rho(\mathbf{z})\left[2 k-k^{2}\right]^{m},
$$

for some positive integers $\rho(\mathbf{z}), m$. Hence the matrix $B_{n}(\mathbf{z})$ is invertible when $k>2$.

Proof. The proof is by induction. Assume $n=2$, then the determinant of $B_{2}$ is

$$
\operatorname{det}\left(B_{2}\right)=i\left|\begin{array}{ll}
-c_{2}+\bar{d} & d-\bar{c}_{2} \\
-c_{2}-\bar{d} & d+\bar{c}_{2}
\end{array}\right|=2 i|d|^{2}-2 i\left|c_{2}\right|^{2} .
$$

Now we assume that every matrix $B_{n-1}$ as above, in $n-1$ variables, satisfies $\operatorname{det}\left(B_{n-1}\right)=(2 i)^{n-2} \prod_{j=2}^{n-1}\left[|d|^{2}-\left|c_{j}\right|^{2}\right]$, and we let $B_{n}$ be

$$
B_{n}=\left(\begin{array}{ccccccc}
-c_{2}+\bar{d} & d-\bar{c}_{2} & 0 & 0 & \ldots & 0 & 0 \\
-i c_{2}-i \bar{d} & i d+i \bar{c}_{2} & 0 & 0 & \ldots & 0 & 0 \\
0 & 0 & -c_{3}+\bar{d} & d-\bar{c}_{3} & \ldots & 0 & 0 \\
0 & 0 & -i c_{3}-i \bar{d} & i d+i \bar{c}_{3} & \ldots & 0 & 0 \\
\vdots & \vdots & \vdots & \vdots & \ddots & \vdots & \vdots \\
0 & 0 & 0 & 0 & \ldots & -c_{n}+\bar{d} & d-\bar{c}_{n} \\
0 & 0 & 0 & 0 & \ldots & -i c_{n}-i \bar{d} & i d+i \bar{c}_{n}
\end{array}\right)
$$

Using the last two columns to evaluate the determinant we get:

$$
\operatorname{det}\left(B_{n}\right)=2 i\left[|d|^{2}-\left|c_{n}\right|^{2}\right] \operatorname{det}\left(B_{n-1}\right),
$$

where

$$
B_{n-1}=\left(\begin{array}{ccccccc}
-c_{2}+\bar{d} & d-\bar{c}_{2} & 0 & 0 & \ldots & 0 & 0 \\
-i c_{2}-i \bar{d} & i d+i \bar{c}_{2} & 0 & 0 & \ldots & 0 & 0 \\
0 & 0 & -c_{3}+\bar{d} & d-\bar{c}_{3} & \ldots & 0 & 0 \\
0 & 0 & -i c_{3}-i \bar{d} & i d+i \bar{c}_{3} & \ldots & 0 & 0 \\
\vdots & \vdots & \vdots & \vdots & \ddots & \vdots & \vdots \\
0 & 0 & 0 & 0 & \ldots & -c_{n-1}+\bar{d} & d-\bar{c}_{n-1} \\
0 & 0 & 0 & 0 & \ldots & -i c_{n-1}-i \bar{d} & i d+i \bar{c}_{n-1}
\end{array}\right)
$$

Then the induction hypothesis implies,

$$
\operatorname{det}\left(B_{n}\right)=2 i\left[|d|^{2}-\left|c_{n}\right|^{2}\right](2 i)^{n-2} \prod_{j=2}^{n-1}\left[|d|^{2}-\left|c_{j}\right|^{2}\right]=(2 i)^{n-1} \prod_{j=2}^{n}\left[|d|^{2}-\left|c_{j}\right|^{2}\right],
$$


proving the first statement in the lemma.

Now choose the indices $\{1, \cdots, n\}$ appropriately, so that $z_{1}, z_{2}, \ldots, z_{m} \neq 0$ and $z_{m+1}=\cdots=z_{n}=0$, for some $m \geq 1$. Notice that $c_{j}(\mathbf{z})=0$ for all $j>m$, and $d(\mathbf{z}) \neq 0$, so we have:

$$
\operatorname{det}\left(B_{n}\right)=(2 i)^{n-1}|d|^{2(n-m)} \prod_{j=2}^{m}\left[|d|^{2}-\left|c_{j}\right|^{2}\right] .
$$

Recall the points of $M$ satisfy the equation 4, that can be seen in norms as $\left|\lambda_{1}\right|\left|z_{1}\right|^{k-2}=\left|\lambda_{j}\right|\left|z_{j}\right|^{k-2}$ for all $j$. Hence:

$$
\begin{aligned}
|d|^{2}-\left|c_{j}\right|^{2} & =\left|\lambda_{1}\right|^{2}\left|z_{1}\right|^{2(k-1)}-(k-1)^{2}\left|\lambda_{1}\right|^{2}\left|z_{1}\right|^{2(k-2)}\left|z_{1}\right|^{2} \\
& =\left|\lambda_{1}\right|^{2}\left|z_{1}\right|^{2(k-1)}\left(1-(k-1)^{2}\right) .
\end{aligned}
$$

Therefore the determinant of $B_{n}$ takes the form

$$
\begin{aligned}
\operatorname{det}\left(B_{n}\right) & =(2 i)^{n-1}\left(\left|\lambda_{1}\right|\left|z_{1}\right|^{k-1}\right)^{2(n-m)} \prod_{j=2}^{m}\left|\lambda_{1}\right|^{2}\left|z_{1}\right|^{2(k-1)}\left(2 k-k^{2}\right) \\
& =(2 i)^{n-1}\left|\lambda_{1}\right|^{2 n}\left|z_{1}\right|^{2(k-1) n}\left[2 k-k^{2}\right]^{m}
\end{aligned}
$$

completing the proof of the lemma.

Proposition 6.7. Let $H_{k}^{n}=\lambda_{1} z_{1}^{k}+\lambda_{2} z_{2}^{k}+\cdots+\lambda_{n} z_{n}^{k}$ and $k>2$, then the intersection of $\mathcal{H}_{k}$ and $M^{*}$ is transversal everywhere.

Proof. Assume $z_{1} \neq 0$ and let $\psi_{j}, \phi_{j}$ and $v_{j}$ be as in the proof of Proposition 6.5 for $j=2, \ldots, n$. The vectors $\left\{v_{2}, i v_{2}, \ldots, v_{n}, i v_{n}\right\}$ form a basis of $T_{\mathbf{z}} \mathcal{L}_{\mathbf{z}}$ as a vector space over $\mathbb{R}$. Notice that the leaf $\mathcal{L}_{\mathbf{z}}$ is not transversal to $M^{*}$ at $\mathbf{z}$ if and only if there exists a vector $v$ in $T_{\mathbf{z}} \mathcal{L}_{\mathbf{z}}$ orthogonal to $\operatorname{grad} \phi_{j}$ and $\operatorname{grad} \psi_{j}$ for all $j=2, \ldots, n$. Then:

Let $\bar{\nabla}$ be as in Section 2, so for each $j$ one has $\bar{\nabla} \psi_{j}(\mathbf{z})=\left(\frac{\partial \psi_{j}}{\partial \bar{z}_{1}}, \ldots, \frac{\partial \psi_{j}}{\partial \bar{z}_{n}}\right)$.

$$
2\langle F(\mathbf{z}), \bar{\nabla} \psi(\mathbf{z})\rangle_{\mathbb{C}}=\langle F(\mathbf{z}), \operatorname{grad} \psi(\mathbf{z})\rangle_{\mathbb{R}}-i\langle i F(\mathbf{z}), \operatorname{grad} \psi(\mathbf{z})\rangle_{\mathbb{R}} .
$$

Remember that $v_{j}$ belongs to $T_{\mathbf{z}} M^{*}$ if and only if the products $\left\langle v_{j}, \operatorname{grad} \psi_{l}\right\rangle_{\mathbb{R}}$ and $\left\langle v_{j}, \operatorname{grad} \phi_{l}\right\rangle_{\mathbb{R}}$ are zero for all $l=2, \ldots, n$. In particular we consider the case $l=j$. We set:

$$
\begin{array}{r}
A_{j}:=\left|\lambda_{1}\right|^{2}\left|z_{1}\right|^{2 k-2}+\left|\lambda_{j}\right|^{2}\left|z_{j}\right|^{2 k-2}, \\
B_{j}:=(k-1)\left[\lambda_{1} \lambda_{j} z_{j}^{k-1} \bar{z}_{j} z_{1}^{k-2}+\lambda_{1} \lambda_{j} z_{1}^{k-1} \bar{z}_{1} z_{j}^{k-2}\right] .
\end{array}
$$

Then:

$$
\left\langle v_{j}, \bar{\nabla} \psi_{j}\right\rangle_{\mathbb{C}}=-A_{j}+B_{j} \quad \text { and } \quad\left\langle v_{j}, \bar{\nabla} \phi_{j}\right\rangle_{\mathbb{C}}=i A_{j}+i B_{j}
$$

Hence:

$$
\left\langle v_{j}, \operatorname{grad} \psi_{j}\right\rangle_{\mathbb{R}}=-2 A_{j}+2 \operatorname{Re} B_{j} \quad, \quad\left\langle i v_{j}, \operatorname{grad} \psi_{j}\right\rangle_{\mathbb{R}}=-2 \operatorname{Im} B_{j},
$$




$$
\left\langle v_{j}, \operatorname{grad} \phi_{j}\right\rangle_{\mathbb{R}}=-2 \operatorname{Im} B_{j} \quad \text { and } \quad\left\langle i v_{j}, \operatorname{grad} \phi_{j}\right\rangle_{\mathbb{R}}=2 A_{j}+2 \operatorname{Re} B_{j} .
$$

Now, using the equation $\left|\lambda_{1}\right|\left|z_{1}\right|^{k-2}=\left|\lambda_{j}\right|\left|z_{j}\right|^{k-2}$ and the polar decomposition of $B_{j}$ we get:

$$
\begin{aligned}
B_{j} & =(k-1)\left[\left|\lambda_{1}\right|\left|\lambda_{j}\right|\left|z_{j}\right|^{k}\left|z_{1}\right|^{k-2}+\left|\lambda_{1}\right|\left|\lambda_{j}\right|\left|z_{1}\right|^{k}\left|z_{j}\right|^{k-2}\right] e^{i\left(\theta_{\lambda_{1}}+\theta_{\lambda_{j}}\right)} e^{i(k-2)\left(\theta_{1}+\theta_{j}\right)} \\
& =(k-1)\left[\left|\lambda_{j}\right|^{2}\left|z_{j}\right|^{2 k-2}+\left|\lambda_{1}\right|^{2}\left|z_{1}\right|^{2 k-2}\right] e^{i\left(\theta_{\lambda_{1}}+\theta_{\lambda_{j}}\right)} e^{i(k-2)\left(\theta_{1}+\theta_{j}\right)}
\end{aligned}
$$

Assume $v_{j}$ is orthogonal to $\operatorname{grad} \phi_{j}$, i.e., $-\operatorname{Im} B_{j}=0$, then

$$
B_{j}= \pm(k-1)\left[\left|\lambda_{j}\right|^{2}\left|z_{j}\right|^{2 k-2}+\left|\lambda_{1}\right|^{2}\left|z_{1}\right|^{2 k-2}\right] .
$$

Therefore,

$$
\left\langle v_{j}, \operatorname{grad} \psi_{j}\right\rangle_{\mathbb{R}}=2\left(\left|\lambda_{j}\right|^{2}\left|z_{j}\right|^{2 k-2}+\left|\lambda_{1}\right|^{2}\left|z_{1}\right|^{2 k-2}\right)[-1 \pm(k-1)] .
$$

Since $z_{1} \neq 0$, we must have $\left\langle v_{j}, \operatorname{grad} \psi_{j}\right\rangle_{\mathbb{R}} \neq 0$. Similar computations work also for $i v_{j}(\mathbf{z})$. Hence we get that, for all $j$, neither $v_{j}(\mathbf{z})$ nor $i v_{j}(\mathbf{z})$ are in $T_{\mathbf{z}} M$, so we arrive to Proposition 6.7.

To complete the proof of Theorem 6.4 we must show that the polar variety $M$ consists of a finite number of lines through the origin. From Proposition 6.5 we know that $M$ has real dimension 2 , it is smooth away from $\mathbf{0}$ and it is analytic at $\mathbf{0}$. The result will be proved if we show that $M$ is a union of complex lines. This is a consequence of the fact that $M$ is defined as the zero set of polynomials of the form $P_{j, l}(\mathbf{z}):=\frac{\partial h_{k}}{\partial z_{j}} \bar{z}_{l}-\frac{\partial h_{k}}{\partial z_{l}} \bar{z}_{j}$, with $\frac{\partial h_{k}}{\partial z_{j}}$ homogeneous (in the complex sense) of degree $k-1$.

Now consider an arbitrary homogeneous polynomial $h$ of degree $k$ in $n$ complex variables, with a unique critical point at $\mathbf{0} \in \mathbb{C}^{n}$, and its corresponding foliation $\mathcal{H}$. Let $\mathcal{C}$ be the space of coefficients of homogeneous polynomials of degree $k$ in $n$ complex variables. The general homogeneous equation of degree $k$ in $n$ variables is:

$$
\sum_{\alpha_{1}+\cdots+\alpha_{n}=k} a_{\alpha_{1}, \cdots, \alpha_{n}} z_{1}^{\alpha_{1}} \ldots z_{n}^{\alpha_{n}} .
$$

The polynomials defining hypersurfaces in $\mathbb{C}^{n}$ with non-isolated singularities correspond to a Zariski closed subset of $\mathcal{C}$. Hence its complement $\Omega$ is connected, and therefore every homogeneous polynomial of degree $k$ in $n$ complex variables with a unique critical point at $\mathbf{0} \in \mathbb{C}^{n}$, is isotopic to a Fermat polynomial $H_{k}^{n}:=z_{1}^{k}+\ldots+z_{n}^{k}$. Then there exists an isotopy $\mathcal{I}$ of $\mathbb{C}^{n}$, fixing the origin 0, carrying the foliation $\mathcal{H}$ of $h$ into the foliation $\mathcal{H}_{k}$ of $H_{k}^{n}$, which carries the Morse structure of the quadratic form $Q$, by Theorem 6.4. We obtain

Corollary 6.8. Let $\mathcal{H}$ be a codimension 1, homogeneous holomorphic foliation germ in $\mathbb{C}^{n}$. Then $\mathcal{H}$ is isotopic to the foliation $\mathcal{H}_{k}$ of $H_{k}^{n}$, and therefore carries a Morse structure compatible with a Morse function isotopic to the quadratic form $Q$. 
The results in this section, together with the aforementioned theorem of Ito-Scardua and other known facts, suggest the following:

Conjecture. Let $\mathcal{F}$ be a holomorphic foliation in an open neighbourhood $\mathcal{U}$ of $\mathbf{0} \in \mathbb{C}^{n}$, singular at $\mathbf{0}$. Then there is an open set $\mathcal{G}$ in the space of all Morse functions with a critical point at $\mathbf{0}$ with Morse index 0 , such that for every $g \in \mathcal{G}$, the contacts of $\mathcal{F}$ with the fibers of $g$ in a neighbourhood of $\mathbf{0}$ are all non-degenerate.

Acknowledgement. The authors are grateful to professors Alberto Verjovsky, Xavier Gómez-Mont, César Camacho, Bruno Scardua, Paulo Sad and Bernard Teissier, for very helpful and fruitful conversations.

\section{References}

[1] A. Andreotti, Th. Frankel. The Lefschetz theorem on hyperplane sections. Ann. of Math. (2), vol. 69 (1959), 713-717.

[2] V. I. Arnold. Remarks on singularities of finite codimension in complex dynamical systems. Funct. Anal. Appl. 3 (1969), 1-5.

[3] P. Baum and R. Bott, Singularities of holomorphic foliations. J. Differential Geom. 7 (1972), 279-342.

[4] A. Bodin, A. Pichon, J. Seade. Milnor fibrations of meromorphic functions. J. Lond. Math. Soc., II. Ser. 80, No. 2, 311-325 (2009).

[5] C. Camacho, N. Kuiper, J. Palis. The topology of holomorphic flows with singularity. Publ. Math. IHES 48 (1978), 143-174.

[6] J. L. Cisneros-Molina. Join theorem for polar weighted homogeneous singularities. In "Singularities II. Geometric and topological aspects". Proc. Int. Conf. "School and workshop on the geometry and topology of singularities". A. M. S. Contemporary Mathematics 475, 43-59 (2008).

[7] J. L. Cisneros-Molina, J. Seade, J. Snoussi. Milnor fibrations and $d$ regularity for real analytic singularities. Int. J. Math. 21, 419-434 (2010).

[8] S. Gitler, S. López de Medrano. Intersections of Quadrics, Moment-angle Manifolds and Connected Sums Preprint 2010; arXiv:0901.2580.

[9] X. Gómez-Mont, J. Seade, A. Verjovsky. Topology of a holomorphic vector field around an isolated singularity. Funct. Anal. Appl. 27 (1993), 97-103.

[10] A. Haefliger. Deformations of transversely holomorphic flows on spheres and deformations of Hopf manifolds. Comp. Math. 55 (1985), 241-251.

[11] Heisuke Hironaka. Subanalytic sets. In Number theory, algebraic geometry and commutative algebra, in honor of Yasuo Akizuki, pages 453-493. Kinokuniya, Tokyo, 1973. 
[12] T. Ito, B. Scárdua. A non-existence theorem for Morse type holomorphic foliations of codimension one tranverse to spheres. International Journal of Mathematics 21 (4)(2010), 435-452.

[13] T. Ito, B. Scárdua, Yamagishi, Y. Transversality of complex linear distributions with spheres, contact forms and Morse type foliations. J. Geometry and Physics 60 (2010), 1370-1380.

[14] D. T. Lê. Computation of the Milnor number of an isolated singularity of a complete intersection. Funct. Anal. Appl. 8, 127-131 (1974).

[15] D. T. Lê, B. Teissier. Variétés polaires locales et classes de Chern des variétés singulieres. Ann. of Math 114 (1981), 457-491.

[16] S. López de Medrano. Topology of the intersection of quadrics in $R^{n}$. In "Algebraic Topology" (Arcata Ca, 1986), Springer Verlag LNM 1370(1989), pp. 280-292, Springer Verlag.

[17] S. López de Medrano, A. Verjovsky. A new family of complex, compact, nonsymplectic manifolds. Bol. Soc. Brasil. Mat., 28 (1997), 253- 269.

[18] B. Malgrange. Frobenius avec singularités. I. Codimension un. Inst. Hautes Études Sci. Publ. Math. 46 (1976), 163-173.

[19] L. Meersseman. A new geometric construction of compact complex manifolds in any dimension. Math. Ann. 317 (2000), 79-115.

[20] L. Meersseman, A. Verjovsky. Sur les variétés LV-M. In "Singularities II. Geometric and topological aspects". Contemp. Math., 475, Amer. Math. Soc., Providence, RI, 2008, p. 111-134, J. P. Brasselet et al., editors.

[21] J. Milnor. Singular points of complex hypersurfaces. Princeton University Press, Annals of Mathematics Studies, No. 61 (1968).

[22] M. Oka. Non-degenerate mixed functions. Kodai Math. J. 33, 1-62 (2010).

[23] M. Oka. On Mixed Brieskorn Variety Preprint 2009, arXiv:0909.4605.

[24] M. A. S. Ruas, J. Seade, and A. Verjovsky. On real singularities with a Milnor fibration. In "Trends in singularities", Trends Math., pages 191-213. Birkhäuser, Basel, 2002. A. Libgober and M. Tibăr, editors

[25] J. Seade. Open book decompositions associated to holomorphic vector fields. Bol. Soc. Mat. Mexicana (3) Vol. 3, 1997.

[26] J. Seade. On the topology of isolated singularities in analytic spaces, Progress in Mathematics vol. 241. Birkhäuser Verlag, Basel, 2006.

[27] T. Suwa. Indices of vector fields and residues of singular holomorphic foliations. Actualités Mathématiques. Paris: Hermann (1998). 
[28] J.-L. Verdier. Stratifications de Whitney et théorème de Bertini-Sard. Invent. Math., 36:295-312, 1976.

[29] R. Thom. Généralization de la théorie de Morse et variétés feuilletées. Ann. Inst. Fourier (Grenoble) 14 (1964), 173-190.

\section{Beatriz Limón and José Seade:}

Instituto de Matemáticas, Unidad Cuernavaca,

Universidad Nacional Autónoma de México,

Av. Universidad s/n, Lomas de Chamilpa, C.P. 62210,

Cuernavaca, Morelos, México.

email: betlimon@matcuer.unam.mx, jseade@matcuer.unam.mx 